\title{
Estimated Uncertainties in the Idaho National Laboratory Matched- Index-of-Refraction Lower Plenum Experiment
}

Donald M. McEligot Hugh M. Mcllroy, Jr. Ryan C. Johnson

November 2007

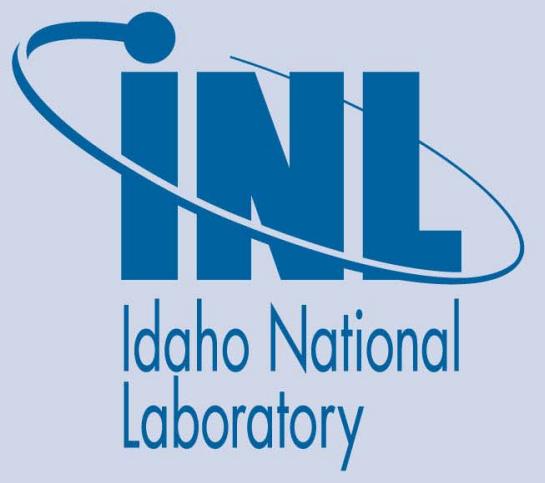

The INL is a U.S. Department of Energy National Laboratory operated by Battelle Energy Alliance 
INL/EXT-07-13539

\title{
Estimated Uncertainties in the Idaho National Laboratory Matched-Index-of-Refraction Lower Plenum Experiment
}

\author{
Donald M. McEligot \\ Hugh M. Mcllroy, Jr. \\ Ryan C. Johnson
}

November 2007

\begin{abstract}
Idaho National Laboratory
Idaho Falls, Idaho 83415
\end{abstract}

Prepared for the

U.S. Department of Energy

Office of Nuclear Energy

Under DOE Idaho Operations Office

Contract DE-AC07-05ID14517 


\section{DISCLAIMER}

This information was prepared as an account of work sponsored by an agency of the U.S. Government. Neither the U.S. Government nor any agency thereof, nor any of their employees, makes any warranty, expressed or implied, or assumes any legal liability or responsibility for the accuracy, completeness, or usefulness, of any information, apparatus, product, or process disclosed, or represents that its use would not infringe privately owned rights. References herein to any specific commercial product, process, or service by trade name, trade mark, manufacturer, or otherwise, does not necessarily constitute or imply its endorsement, recommendation, or favoring by the U.S. Government or any agency thereof. The views and opinions of authors expressed herein do not necessarily state or reflect those of the U.S. Government or any agency thereof. 



\title{
Estimated Uncertainties in the Idaho National Laboratory Matched-Index-of-Refraction Lower Plenum Experiment
}

\author{
INL/EXT-07-13539
}

Revision 0

November 2007

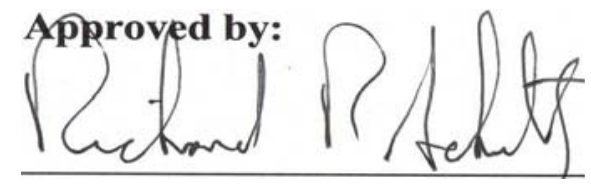

December 19, 2007

Richard R. Schultz, NGNP Methods Program Manager 



\section{ABSTRACT}

The purpose of the fluid dynamics experiments in the MIR (Matched-Index-of-Refraction) flow system at Idaho National Laboratory (INL) is to develop benchmark databases for the assessment of Computational Fluid Dynamics (CFD) solutions of the momentum equations, scalar mixing, and turbulence models for typical Very High Temperature Reactor (VHTR) plenum geometries in the limiting case of negligible buoyancy and constant fluid properties. The experiments use optical techniques, primarily particle image velocimetry (PIV) in the INL MIR flow system. The benefit of the MIR technique is that it permits optical measurements to determine flow characteristics in passages and around objects to be obtained without locating a disturbing transducer in the flow field and without distortion of the optical paths. The objective of the present report is to develop understanding of the magnitudes of experimental uncertainties in the results to be obtained in such experiments. Unheated MIR experiments are first steps when the geometry is complicated. One does not want to use a computational technique, which will not even handle constant properties properly. This report addresses the general background, requirements for benchmark databases, estimation of experimental uncertainties in mean velocities and turbulence quantities, the MIR experiment, PIV uncertainties, positioning uncertainties, and other contributing measurement uncertainties.

The accuracy of the velocity field determination is ultimately limited by the ability of the scattering particles to follow the unsteady motion of the continuous phase. For the particles in the MIR VHTR experiment, the estimated cutoff frequency is about $40 \mathrm{kHz}$. The frequency of shedding from the posts in the MIR model is estimated to be of the order of $1 \mathrm{~Hz}$ and, therefore, the particles should follow the flow.

Based on review of the literature, we estimate that the random uncertainty in measured particle displacement for our system and experiment is about 0.3 pixel for a single realization away from the walls. As an example, we estimated the uncertainties in measurements for the maximum velocity in an inlet jet flow operating at about its minimum flow rate. Consequently, this example would provide worst-case estimates in some senses. For the uncertainty in the mean velocity at this location, one could report $\frac{\delta V_{\text {Mean }}}{V_{\text {Mean }}} \approx 0.6 \%$ (precision) and $0.3 \%$ (bias). The resulting uncertainty in the root-mean-square value of the $u$ fluctuation $\sigma_{\mathrm{u}}$ ' would be about $1 \mathrm{~cm} / \mathrm{s}$. From this example, estimates can be extended to other situations because the particle displacement is given by $\Delta x_{\text {Pixel }}=S \cdot V \cdot \Delta t$, so one can estimate the displacement with another view by taking the ratio of the new $\mathbf{S}$.

With the current MIR model and the 3D PIV system, McIlroy and colleagues examined the effects of sample size for a typical set of measurements. Trends were generally as observed by Uzol and Camci. Preliminary conclusions were that (for this view and processing parameters) about 750 images should be collected to reduce the scatter in mean velocity statistics to between 0.4 and $10 \%$ 
for velocities greater than about $2 \mathrm{~m} / \mathrm{s}$. The trends predicted by an uncertainty analysis are consistent with the observations, but, for the most part, the expected values are less than the scatter observed. Preliminary conclusions from the comparisons are that (1) more samples should be collected than predicted by the uncertainty analysis and (2) longer time intervals, $\Delta t$, should be employed for the low velocity regions.

In addition to the uncertainties in velocity and turbulence measurements by PIV algorithms, other experimental uncertainties must be estimated for proper assessment of proposed CFD codes. The bias uncertainties in fluid properties are about $0.2,2.0$, and $0.02 \%$ for the calibrations of $\rho, v$, and $\mathbf{n}$, respectively. The random uncertainty in kinematic viscosity due to temperature fluctuations of $\pm 0.05^{\circ} \mathrm{C}$ would be $0.2 \%$. The lowest flow rate of the experiment typically is $11.2 \mathrm{gpm}$ for the first jet. At this flow rate, the bias uncertainty would be $0.5 \%$ or about $0.06 \mathrm{gpm}$ and the random uncertainty can be taken as $0.125 \mathrm{gpm}$ or about $1 \%$. The bias uncertainty in the jet Reynolds number would be about $2 \%$, and the random uncertainty would be about $1 \%$. The bias uncertainty is dominated by the uncertainty in the calibration of $v$ and is relatively independent of the uncertainties in flow rate and jet diameter. 


\section{ACKNOWLEDGEMENTS}

Much of the discussion in the introductory sections has been derived from the research of Steve Kline in his honor as leader of the charge. Dr. Steven L. Anderson of LaVision has been extremely helpful in providing information to assist our understanding. Likewise Prof. Barton L. Smith of Utah State University has brought his PIV experience to INL to help develop our expertise in our first PIV experiments with the MIR system.

The study reported was partially supported through the Very High Temperature Reactor (VHTR) program of the Department of Energy (DOE) Office of Nuclear Energy, Science and Technology at INL under DOE Idaho Operations Office Contract DE-AC07-05ID14517. 


\section{CONTENTS}

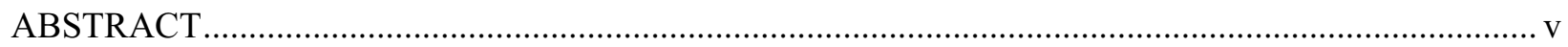

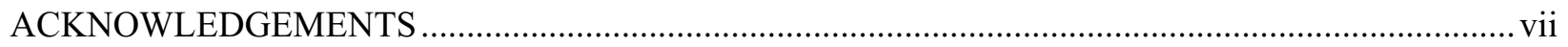

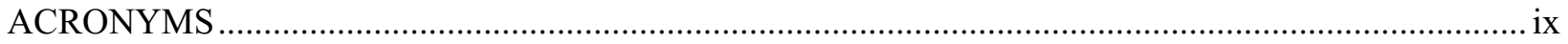

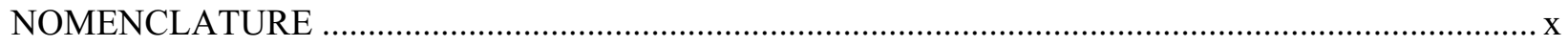

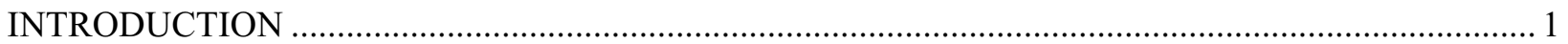

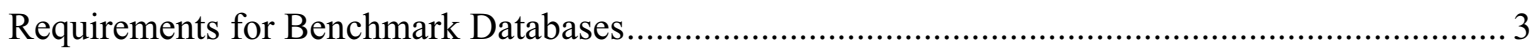

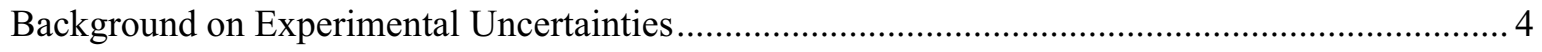

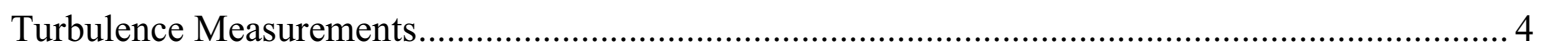

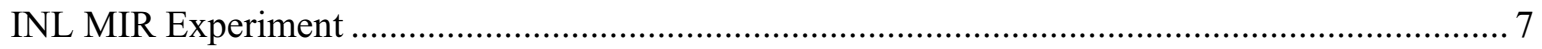

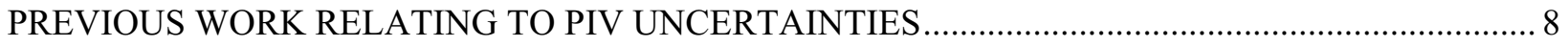

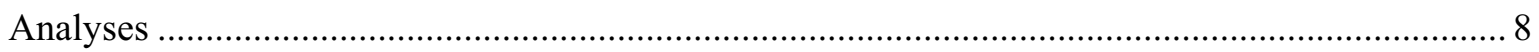

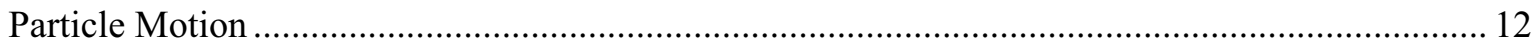

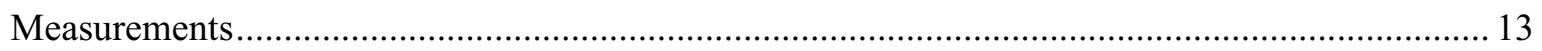

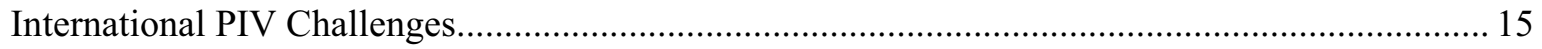

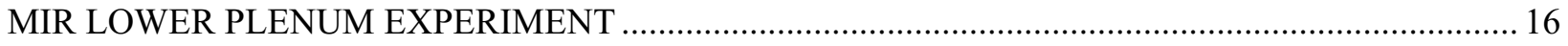

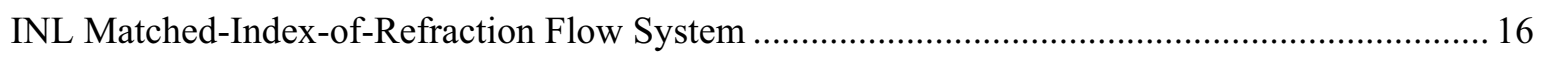

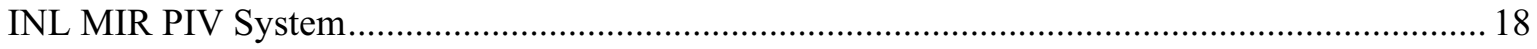

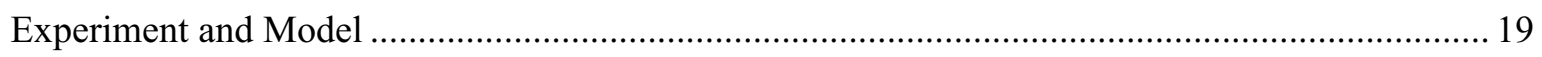

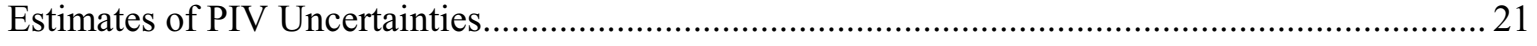

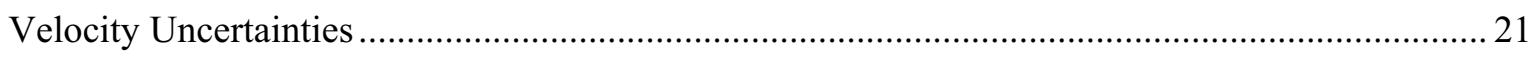

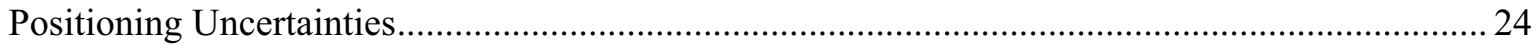

Estimates of Additional Contributing Measurement Uncertainties ............................................ 25

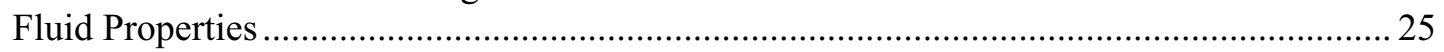

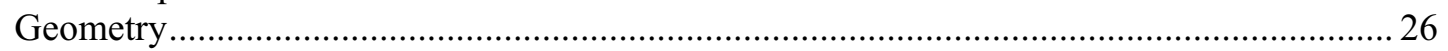

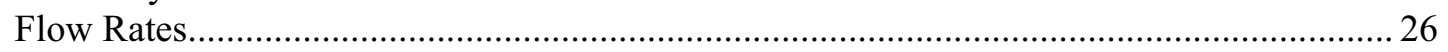

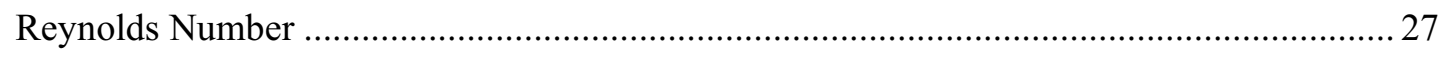

APPLICATION TO MIR LOWER PLENUM EXPERIMENT ............................................................ 28

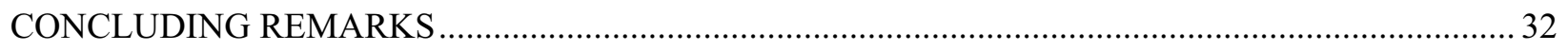

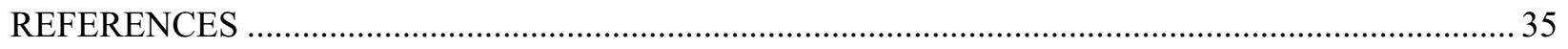

TABLES

Table 1. Typical INL MIR PIV experiment settings. 


\begin{tabular}{ll}
\multicolumn{2}{c}{ ACRONYMS } \\
ANSI & American National Standards Institute \\
ASME & American Society of Mechanical Engineers \\
CC & cross correlation \\
CCD & charge-coupled device \\
CFD & Computational Fluid Dynamics \\
DNS & direct numerical simulations \\
DOE & Department of Energy \\
DOE-EM & Department of Energy Environmental Management \\
FFT & Fast Fourier transform \\
FIND-W & Flow Information Display-Window based \\
INL & Idaho National Laboratory \\
JFE & Journal of Fluids Engineering \\
LDV & laser Doppler velocimeter \\
MIR & Matched-Index-of-Refraction \\
NCC & normalization of cross correlation \\
NERI & National Energy Research Institute \\
NGNP & Next-Generation Nuclear Plant \\
PIPM & particle image pattern matching \\
PIV & particle image velocimetry \\
PTU & Programmable Timing Unit \\
PTV & particle tracking velocimeter \\
rms & root mean square \\
SNR & signal-to-noise ration \\
VHTR & Very High Temperature Reactor \\
&
\end{tabular}




\section{NOMENCLATURE}

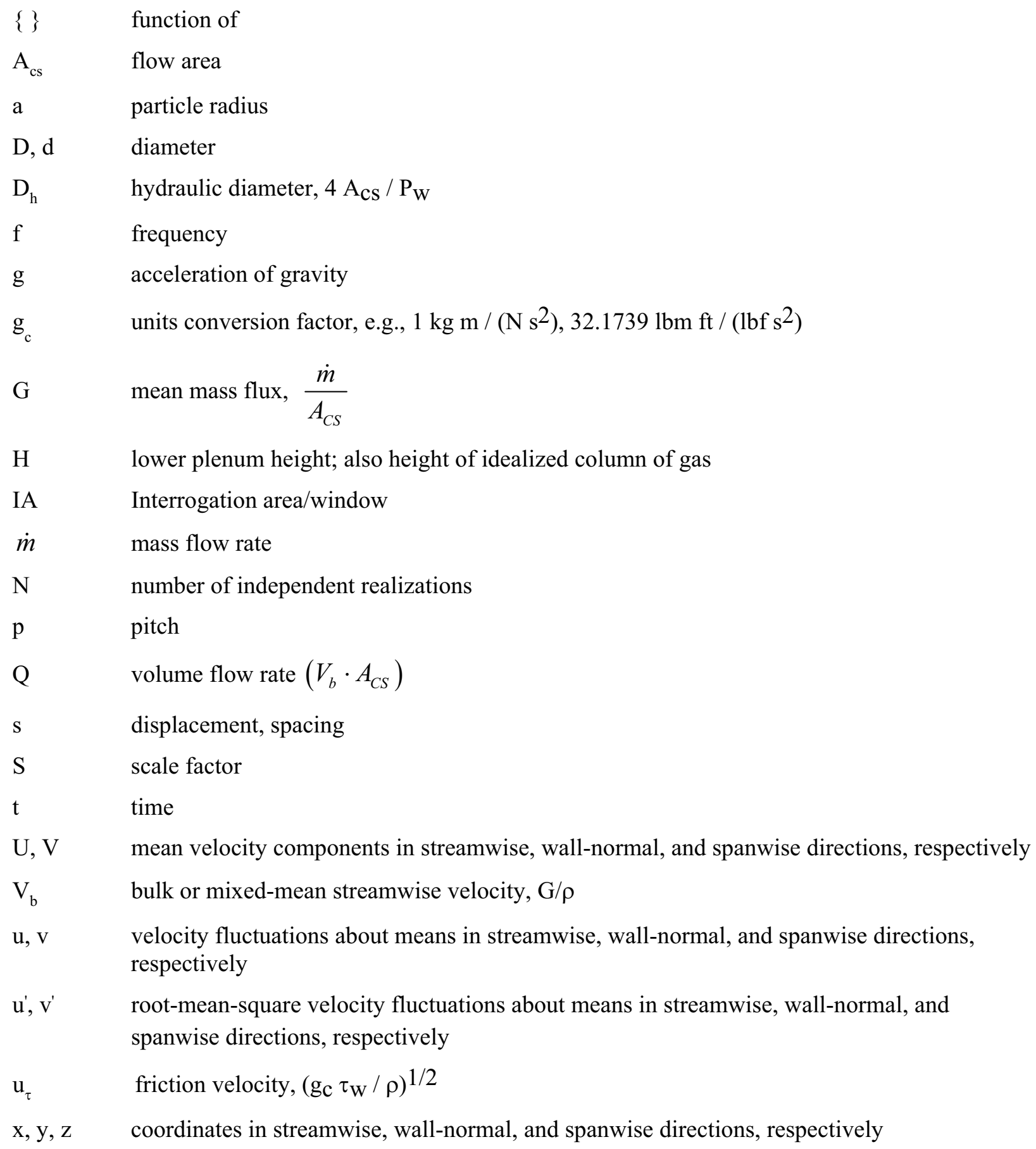




\section{$\underline{\text { Non-dimensional quantities }}$}

Re Reynolds number, $4 \dot{m} / \pi \mathrm{D} \mu ; \mathrm{Re}_{\mathrm{Dh}}$, based on hydraulic diameter, $\mathrm{G}_{\mathrm{h}} / \mu ; \mathrm{Re}_{\mathrm{s}}$, based on channel centerplane, $\mathrm{U}_{\mathrm{CS}}(\mathrm{s} / 2) / \mathrm{v}$

$\mathrm{y}^{+} \quad$ distance from wall to centerplane, centerline, etc., $\mathrm{y}_{\mathrm{c}} \mathrm{u}_{\tau} / \nu$

\section{$\underline{\text { Greek symbols }}$}

$\delta_{-} \quad$ uncertainty of

$\varepsilon \quad$ Stokes number by Mei (1996)

$\mu \quad$ absolute viscosity

$v \quad$ kinematic viscosity, $\mu / \rho$

$\rho \quad$ density

$\sigma \quad$ uncertainty of (quantity indicated by subscript)

$\tau \quad$ shear stress; $\tau_{\mathrm{W}}$, wall shear stress

$\varphi \quad$ correction factor relative to Stokes drag

$\omega \quad$ frequency

\section{$\underline{\text { Subscripts }}$}

b evaluated at bulk or mixed-mean temperature (or enthalpy)

c centerplane, centerline

cs cross section

D based on diameter

$\mathrm{Dh}, \mathrm{D}, \mathrm{h} \quad$ evaluated with hydraulic diameter

f fluid

$i$, in evaluated at inlet, entry, individual realization or discrete time

j jet

p support post, particle

pixel measured in pixels

Quantities for PIV performance analysis are defined in the text. 


\title{
Estimated Uncertainties in the Idaho National Laboratory Matched-Index-of-Refraction Lower Plenum Experiment
}

\author{
INTRODUCTION
}

Unheated Matched-Index-of-Refraction (MIR) experiments are first steps in flow experiments when the geometry is complicated. One does not want to use a computational technique that will not handle constant properties properly. The purpose of the MIR fluid dynamics experiments is to develop benchmark databases for the assessment of Computational Fluid Dynamics (CFD) solutions of the momentum equations, scalar mixing, and turbulence models for typical Very High Temperature Reactor (VHTR) plenum geometries in the limiting case of negligible buoyancy and constant fluid properties. As indicated by scaling studies (McEligot and McCreery, 2004), in normal, full-power operation of a typical VHTR conceptual design, buoyancy influences should be negligible in the lower plenum. The MIR experiment simulates flow features of the paths of jets as they mix in flowing through the array of posts in a lower plenum en route to the single exit duct. The conceptual design for such an experiment is described and the development of the final experimental apparatus is presented by Condie (Condie et al. 2005). Fabrication sketches are provided in Appendix A of their report for the use of CFD analysts wishing to employ the data for assessment of their proposed codes.

In the Idaho National Laboratory (INL) MIR lower plenum experiment, one aspect of the complex flow in a prismatic VHTR is being addressed: flow and thermal mixing in the lower plenum (the "hot streaking" issue). Current prismatic VHTR concepts were examined to identify their proposed flow conditions and geometries over the range from normal operation to decay heat removal in a pressurized cooldown. Approximate analyses were applied to determine key non-dimensional parameters and their magnitudes over this operating range. The flow in the lower plenum can locally be considered to be a situation of multiple jets into a confined crossflow-with obstructions. Flow is expected to be turbulent with momentum-dominated turbulent jets and buoyancy influences are estimated to be negligible in normal, full-power operation. Experiments are needed for assessing the combined features of the lower plenum flows. Missing from the typical jet experiments available are interactions with nearby circular posts and with vertical posts in the vicinity of vertical walls - with near stagnant surroundings at one extreme and significant crossflow at the other.

An objective of the present report is to develop understanding of the magnitudes of experimental uncertainties in the results obtained in such experiments. The experiments use optical techniques, primarily particle image velocimetry (PIV) in the INL MIR flow system (Adrian, 1991; Raffel, Willert and Kompenhans, 1998; Stoots et al., 2001).

As noted by Kline (1985b), "Uncertainty analysis is an essential ingredient in planning, controlling and reporting experiments. The important thing is that a reasonable uncertainty analysis be done." The objective of the present study is to provide a reasonable uncertainty analysis for INL measurements conducted to provide benchmark data for the assessment of computational tools for flow phenomena in the lower plena of proposed VHTRs. These experiments are accomplished in the INL MIR flow system with a LaVision PIV system capable of determining two- and three-dimensional fields of velocities and their related turbulent fluctuations.

A variety of definitions and approaches are employed in obtaining quantitative estimates of uncertainties in experimental results. As emphasized by Kline (1985a), "An uncertainty is not the same as an error." An error in measurement is the difference between the true value and the recorded value. An uncertainty is the possible value that the error might take on in a given measurement and a measurement 
one can estimate. Because the true value is generally unknown in realistic experiments, the values of the errors are also unknown; accordingly, the term "error" is not appropriate for reporting the quantitative accuracy of an experiment. If we keep in mind the usual connotation of the word "error" as a mistake or blunder, we may suppress using the term. On the other hand, Taylor (1997) defines error in a scientific measurement as the inevitable uncertainty that attends all measurements and emphasizes that he uses the two words interchangeably. A discrepancy is the difference between two measured values of the same quantity; it may or may not be significant.

Recent practice in reporting uncertainties categorizes them as precision or bias (Kline, 1985a; Rood and Telionis, 1991; Metzger, 2002). Earlier descriptions included random error or repeatability for precision uncertainty and fixed or systematic error for bias uncertainty. As an example, one can consider measuring a distance with a ruler: typically one can only read the distance reasonably to within about a half-division, so a precision uncertainty would be that range of possible readings about the true value. The difference between the apparent length and true length (e.g., from a calibrated length of negligible bias) indicated by the divisions would contribute to the bias uncertainty and likely would vary depending on the distance from the zero or reference. Due to thermal expansion, this bias uncertainty could vary with temperature so that, if the temperature varies in a random or unknown manner during an experiment, a further contribution to the precision uncertainty could evolve. Calibration can reduce bias uncertainty.

Several useful procedures have been developed for uncertainty analysis and can be used. Procedures for estimating uncertainty in (1) standard tests (e.g., codes for pressure vessel testing) and (2) procedures needed to improve and control accuracy in new experiments of an engineering type typically generate quite different conditions and, hence, need different uncertainty procedures. A single-sample experiment (Kline and McClintock, 1953) is one in which no source of uncertainty has been sampled and no statistical estimates of sample standard deviations can be made. It is appropriate for single-sample experiments and experiments still in the design stage. The approaches of Eisenhart and co-workers at National Institute of Standards and Technology (NIST) are useful for calibration experiments. Those of Abernethy, Benedict, and Dowdell (1985) for the American Society of Mechanical Engineers/American National Standards Institute (ASME/ANSI) standards are pertinent for field, development, and acceptance testing. Kline (Kline and McClintock, 1953) feels that Moffat's methods (Moffat, 1982, 1985, 1988) are useful for reduction of error using uncertainty analysis and, therefore, in research-type experiments.

Uncertainty analysis is an indispensable action that is essential for record experiments and for systematic reduction of errors in experimental work. It is the heart of quality control in experimental work. Kline lists a dozen uses for uncertainty analyses. The following uses are pertinent to the MIR VHTR experiments:

- Identify instruments and procedures that control accuracy.

- Advise early, during the design of an experiment, when an experiment may not meet desired accuracy.

- Provide an appropriate basis to decide whether computations agree with data or lie outside acceptable limits.

- Provide a check against unknowingly taking data in some odd corner of the test-parameter space.

- Provide a basis for deciding whether a closure check has been achieved.

An editorial by Rood and Telionis (1991) provides details on the Journal of Fluids Engineering (JFE) policy on reporting uncertainties in experimental measurements and results, along with some background about deliberations, an example, and some recommended references. They ask for information on the precision and bias limits and a brief description of the methods used. The principal authors of the 
statement adopted were H. W. Coleman and W. G. Steele. A copy is available via the ASME website for JFE.

\section{Requirements for Benchmark Databases}

A preliminary list of criteria or requirements for experiment results to be considered as benchmark databases (and their documentation) should include, but not be limited to, the following:

- Objectives of the database and its experiments

- Precise details of geometries and materials employed (as built dimensions)

- Details of the instrumentation and measuring/sampling techniques

- Sizes of measuring control volumes, sensors and probes, light sheet thicknesses, etc.

- Inlet flow conditions including turbulence distributions, heat loss calibrations, etc.

- Determination of desired fields in the region(s) of interest

- Tabular presentation of the data

- Analyses of the experimental uncertainties of all quantities involved

- Electronic storage of information in a format useful to CFD analysts in multiple forms

- Appropriate graphical representation of key results

- Careful, readable documentation of details

- Comparison to calculations before release

- Web accessible and controlled data

- Long-term point-of-contact.

For assessment of CFD codes predicting turbulent flow, one typically is interested in determining the velocity components and their statistics as functions of position. In some cases, mean statistics will be adequate and, in others, instantaneous values will be desired. In either case, knowledge of uncertainties in the instantaneous quantities will be necessary to perform a reasonable uncertainty analysis. Various integral quantities, such as pressure drops, skin friction coefficients, momentum thicknesses, and heat transfer rates, may also be desirable for overall checks. The code analyst will need descriptions of inlet quantities to specify his/her boundary conditions in a meaningful manner. Whether a transient or steadystate experiment, transient temperatures must be measured and recorded for fluid mechanics experiments in order to deduce the fluid properties, such as density and viscosity, and their random or systematic variations. Transient mass flow rates should also be measured and recorded to check the measured velocity distributions at the inlets and to apply overall mass balances for local and exit flow rates. If the analyst is to conduct a meaningful simulation and comparison, he needs to know the geometry of the experiment precisely. The positions of the measurements must be related to the model geometry.

For proper benchmark databases, the experimental uncertainties of all the measured quantities and their propagation into the results must be estimated quantitatively. In a complicated experiment, such as the MIR lower plenum study, some of these experimental uncertainties can be expected to vary significantly with position (as the local velocities vary).

As a partial tabulation, estimated experimental uncertainties that are expected to be required include, but are not limited to, those for the following quantities:

- Model dimensions 
- Measurement positions relative to the model

- Instantaneous and mean velocity components and their mean statistics, such as Reynolds stresses

- Transient mass flow rates (and the quantities necessary to deduce them)

- Transient temperatures

- Pressure differences, if feasible

- Pertinent instrument characteristics, e.g., laser sheet thickness.

\section{Background on Experimental Uncertainties}

The 1983 Symposium provides comprehensive presentations and discussions of uncertainty procedures and opinions and, therefore, are recommended as a useful introduction. The key papers, discussions, and closure were published in the JFE in its June 1985 issue, on pages 153 through 182. Kline (1985b) also recommends a text by Taylor (1982) for learning more about uncertainty analysis.

A key reference for reporting results of thermal fluid dynamics experiments is the paper by Kline and McClintock (1953) on describing uncertainties in single-sample experiments because many experiments are not repeated multiple times due to cost and/or time constraints. Useful extensions to this reference have been provided by Moffat $(1982,1988)$ and the benefits of application of uncertainty analysis during experiment planning have been championed by Smith and Wehofer (1985) and Moffat (1985).

Relationships to calculate propagation of individual uncertainties into the reported results and some examples are presented by Kline and McClintock, Kline ([1985a], Taylor 1982, 1997) and others. Assumptions that the uncertainties considered are random and independent are typically implied if not mentioned. Loosely stated, when the operation is addition and/or subtraction, the uncertainty in the absolute value of the result is given by root mean square ( $\mathrm{rms})$ combination of the absolute uncertainties of the quantities involved. For multiplication and/or division, the percentage uncertainty (or fractional uncertainty [Taylor, 1997]) of the result is obtained from rms combination of the percentage uncertainties of the quantities involved. If the calculation is a power law (e.g., $r=u^{n}$ ), the percentage uncertainty of the result is provided by the exponent multiplied by the percentage uncertainty of the quantity; consequently, for a square root, it becomes half the percentage uncertainty of the quantity. In the situation of a complicated data reduction procedure accomplished via a computer program, sensitivities can be determined by repeated application of the calculations with perturbation of the input data as suggested by Moffat (1982).

Normally, rms combination will be used separately for the precision and bias uncertainties at each step up to the final step, where either rms or linear combination is acceptable, provided one states the basis. The Journal of Fluids Engineering (Rood and Telionis, 1991) suggests that the final uncertainty be calculated as the rms combination of the precision and bias uncertainties.

\section{Turbulence Measurements}

In her thesis, Metzger (2002) provides a useful discussion of approaches to uncertainty analyses in general and to turbulence quantities in particular. She describes three different approaches, ranging from a worst case to neglect of bias uncertainties, and derives equations for the propagation of uncertainties for scalar transport. Relations for mean values, fluctuating quantities, derivatives, dissipation, and products are developed. In her case, any given instantaneous measurement has an associated uncertainty, primarily due to uncertainties in the calibration parameters and baseline instrumentation noise. She notes that uncertainties in the calibration parameters affect each measurement equivalently and, therefore, introduce bias uncertainties into individual measurements. 
The instantaneous velocity components are typically represented as $\mathbf{U}+\mathbf{u}$, where $\mathbf{U}$ is its mean value and $\mathbf{u}$ is the fluctuation about the mean. The mean value will suffer both systematic and random uncertainties. However, calculation of the fluctuation as the difference from the mean leaves the systematic (bias) uncertainty affecting the mean value; however, normally only the random uncertainties are associated with the fluctuations about it.

Metzger suggests that the bias uncertainty in the mean value of a time series is given by

$$
b=\frac{1}{N} \sum b_{i}
$$

where

$$
\begin{aligned}
& \mathbf{b}_{\mathrm{i}}=\text { bias uncertainties at each time, and } \\
& \mathbf{N}=\text { total number of independent samples (Metzger Eqn. 4.49). }
\end{aligned}
$$

The random uncertainty of the mean is

$\sigma_{r}=\sqrt{\frac{\sigma^{2}}{N}}$ with $\sigma^{2}=\frac{1}{N} \sum \sigma_{i}^{2}$,

where

$$
\sigma_{\mathrm{i}}=\text { random uncertainty at each time. }
$$

If $\sigma_{\mathbf{i}}$ is taken as the same for all samples, the random uncertainty in the mean becomes $\frac{\sigma_{i}}{\sqrt{N}}$.

The uncertainty in the mean-square value of a fluctuating quantity, such as the Reynolds normal stress $\left(\mathbf{u}^{\prime}\right)^{\mathbf{2}}\left(\right.$ or $\left.\left(\mathbf{v}^{\prime}\right)^{\mathbf{2}}\right)$, can be estimated to be

$$
\sigma_{\left(u^{\prime}\right)^{2}} \approx \sqrt{\left(u^{\prime}\right)^{2}} \cdot \frac{\sigma_{u}}{\sqrt{\frac{N}{2}}},
$$

where

$\sigma_{\mathrm{u}}=$ random uncertainty in $\mathbf{u}$,

the quantity $\mathbf{u}^{\prime}=$ root-mean-square value of the fluctuations, and

$\mathbf{N}=$ number of independent realizations (Metzger, 2002).

As shown here, the units of measurement would be $\mathrm{m}^{2} / \mathrm{s}^{2}$. This approximation can be rearranged to provide the estimated experimental uncertainty in $\mathbf{u}^{\prime}$ as

$$
\sigma_{u^{\prime}} \approx \frac{\frac{\sigma_{u}}{2}}{\sqrt{\frac{N}{2}}}
$$


and, correspondingly, for $\mathbf{v}^{\prime}$ with units of $\mathrm{m} / \mathrm{s}$.

Typical relations for estimating the propagation of uncertainties (rms combination) are based on the assumption that the quantities are random and independent. In particular, for a turbulent flow, $\mathrm{N}$ represents the number of independent realizations of the quantity. To follow the temporal details of a flow, rapid sampling is desirable; that is, one would prefer the sampling interval to be considerably shorter than the time scale of the flow investigated. However, this approach will not give independent realizations for calculating mean statistics. For example, velocity measurements at nearby points or times in a given eddy will not be independent of each other. Consequently, a typical rule-of-thumb is to require that the counting of independent realizations be based on the number of separate eddies occurring. In some situations this determination can be approximated analytically, whereas in others examination of convergence of the mean statistics of the actual data will be required.

The number of realizations can be estimated from the duration of the measurements divided by a characteristic time deduced from a characteristic length (possibly an eddy size in a turbulent boundary layer) and a characteristic velocity. For a channel, the characteristic length might be considered to be its spacing, depth, or length, with the last being the most conservative (i.e., the fewest realizations). In earlier studies with the channel used by McEligot and Eckelmann (2006), Kreplin found that, in order to achieve a reproducible mean value of the velocity within 1\%, measuring times of 20 to 30 minutes were necessary at $\operatorname{Re}_{\mathrm{s}} \approx 7700$ and 15 to 40 minutes at $\mathrm{Re}_{\mathrm{s}} \approx 4700$ (Kreplin, 1973, 1976; Eckelmann personal communication, 2005). Kreplin's observations are consistent with the selection of channel length as the characteristic dimension. For their estimates of uncertainties in $\mathbf{u}^{\prime}$ and $\mathbf{v}^{\mathbf{}}$, McEligot and Eckelmann employed the centerline velocity and the conservative choice of channel length. Consequently, for their 200,000 samples over 4,000 seconds, the number of independent realizations was estimated to be about 70 to 90, depending on the experimental run (McEligot and Eckelmann, 2006).

If the flow phenomena involve the shedding of eddies from a post or rod, the order-of-magnitude of the time between successive eddies may be estimated from their Strouhal frequency, typically around 0.2. If another periodic phenomenon is significant, its period could be important as well. The blade-passing frequency of a circulating pump may be faster or slower than the nominal Strouhal frequency for the mean flow. Turbulent eddies in the shear layer around a cylinder will likely be of smaller scale and, thus, higher frequencies than the cylinder eddies. If these phenomena cannot be separated in the data acquisition or reduction (for example, by a phase-averaging approach), some conservative estimate will be needed for the required sampling duration to ensure sufficient independent realizations. If, by chance, the instrument sampling frequency is the same as the frequency of the periodic flow phenomenon, misleading results could be obtained for mean values and their rms fluctuations.

For two well-defined frequencies, the beat phenomenon might be considered (Halliday and Resnick, 1970). The frequency at which the combined signal repeats is half the difference in frequencies of the basic signals. Thus, for signals which have approximately the same frequencies, the duration to obtain a given number of repetitions of the combined signal can become long even though the individual frequencies are high.

In the processing of PIV data, missing velocity vectors may occur near a surface or from a poor seeding situation. While the calculation of the mean statistics treats only the valid vectors, the total number of realizations at such points will be less than the number of frame pairs analyzed. With the INL LaVision system, the operator can specify a minimum number of valid vectors at each node point in order for statistics to be calculated there (Anderson, e-mail, 13 March 2006). Where holes appear in the tabulated statistics, one knows that less than the specified number of vectors was found at those points. Thus, one can ensure a minimum number of realizations for the reported data and their statistics (provided later post processing does not fill the holes somehow). Whether or not these realizations are independent depends on the sampling intervals and the time scales of the flow phenomena. 


\section{INL MIR Experiment}

The first experiment addresses flow in the region on the central plane away from the plenum outlet duct. Conceptually, there is no significant flow from the outer reflector (except some bypass flow between the prismatic blocks) and the dominant flow comes from the jets issuing from short coolant ducts at the corners of the hexagonal blocks (corner channels). The main plenum crossflow evolves from these jets in the active core and then passes below the central reflector, which lacks the corner coolant ducts (i.e., no additional jet inflow).

The model is scaled to the geometric dimensions of the NGNP Point Design (MacDonald et al., 2003) as an example of a typical prismatic VHTR. With a desired height-to-diameter ratio of about seven, optical constraints led to a post diameter $\mathrm{D}_{\mathrm{p}}$ of $1.25 \mathrm{in}$. $(31.8 \mathrm{~mm})$. The model dimensions are post-height-to-diameter ratio $(H / D)=6.85$, equilateral triangular pitch-to-post-diameter ratio $(p / D)=1.70$ and a diameter ratio $\left(\mathrm{D}_{\mathrm{j}} / \mathrm{D}\right)$ of 0.696 for the jet inlet ducts. The post diameter is $1.250 \pm 0.002 \mathrm{in}$. $(3.175 \pm 0.005 \mathrm{~cm})$. The model consists of a row of full circular posts along its centerplane with half-posts on the two parallel walls to induce flow features somewhat comparable to those expected from the staggered adjacent rows of posts in the reactor design. The posts and half-posts form an equilateral triangular pattern, supporting a core consisting of hexagonal blocks.

The target temperature for matching the refractive indices at $532 \mathrm{~nm}$ is $23.3^{\circ} \mathrm{C}$ (McIlroy, e-mail, 29 March 2006). At this temperature oil properties are density $\rho \approx 831.2 \mathrm{~kg} / \mathrm{m}^{3}\left(51.89 \mathrm{lb} / \mathrm{ft}^{3}\right)$ and kinematic viscosity $v \approx 14.12 \mathrm{cS}=14.12 \times 10^{-6} \mathrm{~m}^{2} / \mathrm{s}\left(0.5470 \mathrm{ft}^{2} / \mathrm{hr}\right)$.

The lowest flow rate likely to occur in an MIR experiment with the lower plenum model (Condie et al., 2005) would be for a single jet operating at a Reynolds number Rejet of about 4,000 without an imposed cross flow. Other cases can be scaled from this situation. The mass flow rate would be about $0.8 \mathrm{~kg} / \mathrm{s}(6,500 \mathrm{lb} / \mathrm{hr})$ with a volume flow rate of about $3.5 \mathrm{~m}^{3} / \mathrm{hr}(16 \mathrm{gpm})$. The jet bulk velocity would be about $2.6 \mathrm{~m} / \mathrm{s}(8.4 \mathrm{ft} / \mathrm{s})$ and the average bulk velocity $\mathrm{V}_{\mathrm{Max}}$ at the minimum flow area in the model array would be about $0.20 \mathrm{~m} / \mathrm{s}(0.67 \mathrm{ft} / \mathrm{s})$. Based on the definition of Zukauskas (1972), the Reynolds number for flow across a tube array would be $\left(\frac{V_{M a x} D}{v}\right)$, about 460 where $\mathrm{D}$ is the tube (post) diameter.

In the MIR lower plenum experiments, sampling in the three-dimensional mode is 2 to $3 \mathrm{~Hz}$. The minimum flow rate planned is for a single-jet experiment with $\mathrm{Re}_{\mathrm{Jet}}$ about 4,000. In this case, as noted above, the streamwise bulk velocity in the test section would be about $0.20 \mathrm{~m} / \mathrm{s}(0.67 \mathrm{ft} / \mathrm{s})$; thus, eddy shedding from the posts (if it exists) would be expected to be at the order of $1 \mathrm{~Hz}$. Consequently, the flows in successive frame pairs might not be independent, and additional sampling could be necessary to achieve sufficient numbers of independent realizations for the mean statistics. If there is a beat phenomenon involved as well, the necessary duration of sampling would be extended. With four jets, each operating at $\mathrm{Re}_{j}$ et of 4,000, the flow rate, array velocity, and shedding frequency would be four times higher, and independent realizations might be achieved when sampling at 2 to $3 \mathrm{~Hz}$ (provided there is not a significant beat phenomenon).

In an experiment with features comparable to the MIR lower plenum study, Uzol and Camci (2001) examined the effect of sample size, turbulence intensity and the velocity field on the experimental accuracy of ensemble-averaged PIV measurements in cylinder wakes. The situation gave wide variations in the magnitudes of mean velocity components and high turbulence levels. Randomly selected averages of 5, 10, 25, 50, 100, 500, 750, and 1,000 samples were calculated. Uzol and Camci found that uncertainties in ensemble-averaged data increase with both increasing turbulence levels and decreasing 
velocity magnitudes. From their figures, it appears that about 500 or more samples were needed for convergence of the calculated statistics. Camci (email, 26 January 2006) believes that for 3D-PIV, statistically-stable ensemble averages require at least 1,000 to 1,200 ensembles in their recent phaselocked experiments.

To ensure that sufficient numbers of independent realizations are obtained and the desired accuracy can be achieved, it is recommended that early experiments examine the convergence of the mean statistics versus numbers of sample pairs, as done by Ullum et al. (1998) and by Uzol and Camci (2001).

\section{PREVIOUS WORK RELATING TO PIV UNCERTAINTIES}

Uncertainties in velocity measurements include those introduced during the recording of multiple exposures (such as ones introduced by distortion of the scene being recorded), limited lens and film resolution, three-dimensional effects, bias introduced by large velocity gradients, and inaccuracies due to processing algorithms (Lourenco and Krothapalli, 1995). Guezennec and Kiritsis (1990) addressed the PIV velocity error as functions of image noise level, number of particles, and particle displacement. Willard (1992) examined dependencies on particle density, particle displacement, and velocity gradients.

\section{Analyses}

Many investigators have examined processes and algorithms for estimating the particle displacement in PIV images. In comparisons to the known velocities in synthetic images, the authors can calculate errors (as opposed to uncertainties). These calculated errors give us insight when estimating uncertainties in real data where the actual velocity is not known.

Keane and Adrian $(1990,1992)$ studied performance optimization of 2D-PIV processing for autocorrelation and cross-correlation methods. They indicate that performance of PIV is determined by the spatial resolution, the valid detection rate and the accuracy of the velocity measurements. The detection rate is defined as the number of interrogation spots per unit area of image plane, which produce velocity measurements that satisfy certain detection criteria. Most of the emphasis in their papers is on detection probabilities.

Identified are a number of non-dimensional experiment parameters that affect these measures of performance. Based on analysis and Monte Carlo simulation, Keane and Adrian provide guidelines for selection of these parameters in a given flow field. Pertinent quantities identified include the following:

- $\mathrm{X}$, location in the image plane

- $\mathrm{x}$, location in the fluid

- $\mathrm{u}\{\mathrm{x}\}$, local velocity at center of measurement volume

- M, lens magnification of motion in the image plane relative to the fluid motion, e.g., pixels $/ \mathrm{cm}$

- $\mathrm{C}$, mean concentration of seeding particles, particles/volume

- $\mathrm{d}_{\mathrm{I}}$, size of interrogation spot on image

- $\mathrm{d}_{\tau}$, particle image diameter, e.g., pixels, or particle diameter on image

- $\mathrm{N}_{\mathrm{I}}$, particle image density or mean number of particles in the interrogation spot, particles/spot (or particles/window), determined from $\mathrm{C}$ via $\mathrm{M}$

- $\Delta \mathrm{z}_{\mathrm{o}}$, laser sheet thickness

- $\Delta \mathrm{t}$, time interval between successive images. 
Effects with weak velocity gradients are evaluated by considering a steady uniform velocity field. For strong velocity gradients, the velocity variation is taken to be linear in the instantaneous field.

For the double-pulse autocorrelation technique with single frame recording (Keane and Adrian, 1990), the key non-dimensional parameters to optimize the performance (as defined by the authors) are as follows:

- $\quad \mathrm{N}_{\mathrm{I}}$, particle image density

- $\mathrm{D}_{0}$, signal-to-noise ratio in the resulting correlation function

- $|\Delta \mathrm{X}| / \mathrm{d}_{\mathrm{I}}\left(\right.$ or $\left.\mathrm{M}\left|\mathrm{u}_{\mathrm{I}}\right| \Delta \mathrm{t} / \mathrm{d}_{\mathrm{I}}\right)$, mean in-plane displacement relative to spot size

- $|\Delta \mathrm{u}| /\left|\mathrm{u}_{\mathrm{I}}\right|$, amplitude of velocity variation across interrogation region due to velocity gradient

- $\quad \mathrm{M}|\Delta \mathrm{u}| \Delta \mathrm{t} / \mathrm{d}_{\tau}$, amplitude of velocity variation across interrogation region due to velocity gradient, relative to particle diameter, in terms of differing displacements

- $\mathrm{d}_{\tau} / \mathrm{d}_{\mathrm{I}}$, particle image diameter relative to spot size

- $\quad \mathrm{w} \Delta \mathrm{t} / \Delta \mathrm{z}_{0}\left(=\Delta \mathrm{z} / \Delta \mathrm{z}_{0}\right)$, out-of-plane displacement.

The processing technique considered is sensitive to the percentage of potential particle pairs that remain in the interrogation region during the time interval. Particle motion between exposures can cause some particles to leave the interrogation volume so the maximum number of true image pairs is less than the possible number and the amplitude of the response is reduced. This situation is called the loss-of-pairs effect by the authors; it is a consequence of the out-of-plane motion $\left(\Delta \mathrm{z} / \Delta \mathrm{z}_{0}\right)$ and the in-plane motion $\left(\Delta X / d_{\mathrm{I}}\right)$. Keane and Adrian (1990) recommend constraining both of these quantities to less than $25 \%$.

Increasing the image density, $\mathrm{N}_{\mathrm{I}}$, generally increases the detection probability and valid detection probability. The authors suggest that $\mathrm{N}_{\mathrm{I}}$ be greater than 15 and that the in-plane displacement $|\Delta X| / \mathrm{d}_{\mathrm{I}}$ be 0.25 or less. Keane and Adrian (1990) recommend adjusting the time interval between light pulses, $\Delta t$, to satisfy this criterion.

Gradients affect the processing technique in two ways. One is the relation of the displacement correlation and the mean fluid motion (Adrian, 1988). The second is that gradients diminish the amplitude of the correlation peak and broaden its width. Consequently, results are biased towards velocities lower than that at $\mathrm{x}_{\mathrm{i}}$; this phenomenon is known as gradient bias and is determined by $\mathrm{M} \Delta \mathrm{u} \Delta \mathrm{t} / \mathrm{d}_{\mathrm{I}}$, which is the displacement variation. Detection bias occurs because the variation of displacements from randomly-located particles lowers the probability of detecting the correlation peak within the noise peaks. The reduction is related to $M|\Delta \mathrm{u}| \Delta \mathrm{t} / \mathrm{d}_{\tau}$; thus, the authors suggest minimizing the velocity variation within the interrogation volume by a judicious selection of $\Delta \mathrm{t}$. Further, the broadening of the displacement component leads to increased uncertainty in estimating (mean) velocity from individual realizations.

Keane and Adrian (1990) indicate that high detection probabilities can be achieved if the velocity variation $\mathrm{M}|\Delta \mathrm{u}| \Delta \mathrm{t} / \mathrm{d}_{\tau}$ is less than unity and the displacement $|\Delta \mathrm{X}| / \mathrm{d}_{\mathrm{I}}$ is held below 0.25 , as suggested above. These constraints imply that $(\Delta \mathrm{u} / \mathrm{u})<4 \mathrm{~d}_{\tau} / \mathrm{d}_{\text {I }}$, which can be used to estimate the maximum velocity difference that can be comfortably treated by the correlation technique or to define the diameter needed. Hence, velocity gradients ultimately place a fundamental limit on the measurements that can be made with double-pulsed autocorrelation PIV. 
For this technique, the relative bias $\mathrm{u}_{\mathrm{Meas}} / \mathrm{u}$ due to variation of $\mathrm{u}$ is approximately independent of image displacement and varies approximately linearly with $\mathrm{M}|\Delta \mathrm{u}| \Delta \mathrm{t} / \mathrm{d}_{\mathrm{I}}$. As an example, for the operating parameters of Keane and Adrian (1990) Figure 13, at $\mathrm{M}|\Delta \mathrm{u}| \Delta \mathrm{t} / \mathrm{d}_{\mathrm{I}} \approx 0.05$ the reduction is about $7 \%$. The authors note that the only way to reduce this bias is to reduce the time interval $\Delta \mathrm{t}$, but this approach also decreases the accuracy as $|\Delta \mathrm{X}|$ decreases. The velocity gradient causes random errors in valid measurements due to the random particle locations within the varying velocity field. Consequently, apparent turbulent fluctuations occur in a steady laminar flow. To restrict this variation of $\mathrm{u}_{\text {Meas }}$ about its mean, the previous criterion that $\mathrm{M}|\Delta \mathrm{u}| \Delta \mathrm{t} / \mathrm{d}_{\tau}<1$ is applicable.

The reader is reminded that the previously stated recommendations of Keane and Adrian (1990) pertain to double-pulse planar PIV with analysis by full 2-D spatial autocorrelation.

Keane and Adrian (1992) extended their 1990 study to examine cross-correlation methods of interrogation of successive single-exposure frames used to measure the separation of pairs of particle images between the successive frames. By knowing the extent of image shifting used in a multipleexposure and with a priori knowledge of the mean flow field, the cross-correlation of different-sized interrogation spots with known separation can be optimized in terms of spatial resolution, detection rate, accuracy, and reliability. Again, theoretical and Monte Carlo approaches are employed to examine the mean results for the case of a fluid velocity field with local linear variation.

Keane and Adrian conclude that single- and double-exposure double-pulse PIV systems are superior to double-pulse systems using autocorrelation analysis for a number of reasons. The versatility in selecting size and location of successive interrogation spots permits a higher level of spatial resolution by more efficient pairing of particle images. Spatial resolution can be improved or seeding density can be reduced. Further, in cross-correlation of single-exposure systems, image shifting is not necessary to resolve directional ambiguity. Consequently, the dynamic range of velocity measurements is doubled.

To avoid loss-of-pairs problems, Keane and Adrian suggest increasing the size and light sheet thickness of the second window so that all particle images in the first window are included in the second. Additional non-dimensional parameters appearing in the analysis of double-frame techniques include

$$
\frac{\left|X_{2}-X_{1}\right|}{d_{1}}, \quad \frac{d_{2}}{d_{1}}, \quad \text { and } \frac{\Delta \mathrm{z}_{02}}{\Delta z_{01}},
$$

where the subscripts identify the first and second frames. Recommended values of the non-dimensional operational parameters are approximately the same as for autocorrelation techniques with a slight relaxation of some. Examples are $1<\mathrm{D}_{0}<1.2, \mathrm{M}|\Delta \mathrm{u}| \Delta \mathrm{t} / \mathrm{d}_{\mathrm{I}}<0.03$ and $\left(\mathrm{N}_{\mathrm{I}}\right)_{1}$ greater than seven. These suggestions may depend on the elimination of the loss-of-pairs problem, as recommended above; however, these modifications are not necessarily practical with commercial systems.

For the simulations with known velocity fields, the random errors apparently were determined as the variations - about the deduced mean - in the individual realizations for deducing the mean displacements. These random errors are due to imperfections in the particles, the recording process, electronic noise in camera output, other practical limitations on obtaining particle images and on the interrogation method and peak location technique. Adrian (1991) suggests that it should scale with particle image diameter $\mathrm{d}_{\tau}$ with an approximately linear variation. The constant of proportionality was about 0.07 in the study of Prasad et al. (1992), giving a minimum total error (bias plus random) between 0.1 and 0.2 pixels at $d_{\tau} / d_{\text {pixel }}$ between one and two. 
Lourenco and Krothapalli (1995) compared a number of peak-finding algorithms aimed at identifying the location of the peak in an autocorrelation to within better resolution than an individual pixel. Two methods, Gaussian fit with FFT padding and Whittaker's reconstruction or Cardinal interpolation, were recommended. Experiments with a steady laminar wall jet of air were employed in conjunction with a numerical solution to evaluate PIV measurement of velocities and vorticity (i.e., velocity gradients). Lourenco and Krothapalli show that uncertainties in the measurement of vorticity depend on two components of a different nature. The truncation error of the differencing scheme decreases as the grid size is reduced, while the uncertainty in velocity measurement increases. By comparison to the numerical solution, they showed that individual realizations of the vorticity could have large errors. With a differencing scheme based on Richardson's extrapolation principle, plus least-squares fitting of the velocity data set, they obtained improved agreement of vorticity distributions.

Huang, Dabri, and Gharib (1997) focused on error quantification associated with the processing method in digital PIV. They indicated three forms of errors: outliers, mean-bias errors, and rms errors. Outliers usually appear randomly in direction and amplitude; because these are usually larger than one pixel, they are easy to detect. Factors contributing to outliers are insufficient numbers of particles in the particle image pattern, strong velocity gradients, and strong three-dimensional flow motions. Huang, Dabri, and Gharib (1997) note that Keane and Adrian (1990), Willert (1992), and several others discuss methods to reduce or remove outliers. Huang, Dabri, and Gharib define mean and rms errors in terms of a unique displacement applied to all particles. The mean-bias error is the difference between the known displacement and the mean of the displacements determined at the various interrogation locations. The rms error is then the root-mean-square of those displacements about their mean, which is comparable to a mean velocity and the fluctuations about it in a turbulent flow, except that these PIV errors are the result of random spatial locations at the same instant.

Huang, Dabri, and Gharib (1997) suggest that the existence of a non-zero mean-bias error indicates that the shape of the correlation peak does not agree with the fitted curve in the mean. They note that the rms error may be affected by many factors. In addition to improper particle seeding, strong velocity gradients, and three-dimensional flow, other factors such as camera dark-current noise, non-linear and non-uniform camera response, non-uniformity of illumination, non-uniform reflection of particles at different locations and angles, and introduction of cable noise and digitization error can contribute to the rms error. To combine these effects, Huang, Debri, and Gharib defined image noise as all contributions other than the particle image itself. They then concentrated on the combined contribution of the processing method and camera noise to the mean-bias and rms errors. Processing techniques included cross-correlation (CC) and particle image pattern matching (PIPM), which is based on their directlycomputed normalized correlation function. They concluded that both mean-bias and rms errors were of the order of 0.1 pixels for CC and about 1 order-of-magnitude less for PIPM. Normalization of CC (NCC) improves accuracy without adding much computation effort. However, with the noisy images of a CCD video camera (rather than simulated images) the rms errors of the PIPM and NCC techniques increased to about 0.1 pixels as well.

Westerweel (2000) derived an analytical expression for the error in sub-pixel displacement in terms of source density. In his introductory review he notes that the measurement error can be reduced to 0.04 pixels under controlled circumstances as he and his colleagues have demonstrated experimentally. His analysis indicates that this measurement error could be reduced to about 0.02 pixels under ideal circumstances, which are difficult to achieve. His theory predicts that minimum error is found for a particle-image diameter of about 2 pixels and that the error is proportional to the particle-image diameter. He notes that the range of measurement errors found in actual PIV measurements is about 0.05 to 0.1 pixels.

For the purpose of estimating the uncertainty in the instantaneous velocity determined from a single pair of images, it seems appropriate to consider an rms uncertainty estimated from the mean-bias error 
and the rms error, and one would expect it to be random rather than consistent. However, the presence of a wall and its associated velocity gradients can be expected to introduce steady errors (bias or fixed), which vary spatially relative to the surface.

\section{Particle Motion}

Sridhar and Katz (1995) measured forces acting on bubbles as they are entrained by a vortex. They used triple-exposure PIV, thereby giving velocity and acceleration of the fluid and bubbles simultaneously. In their paper they provide a useful review of data available for solid spheres and, in an appendix, explanation of their uncertainty estimates for velocity, acceleration, and vorticity.

As noted by Melling (1997), the accuracy of the velocity field determination is ultimately limited by the ability of the scattering particles to follow the unsteady motion of the continuous phase. The choice of optimal diameter for seeding particles is a compromise between an adequate tracer response of the particles in the fluid, requiring small diameters, and a high signal-to-noise ratio (SNR) of the scattered light signal, necessitating large diameters. The angular distribution of scattered light in the direction of the camera is complex. However, in general, larger particles will give stronger signals. Melling analyzed the motion of suspended particles in turbulent flows, primarily gases, because choice of particle size is normally more critical for gases than liquids. He examined the case without external forces such as gravity.

Melling used solutions of the equation for unsteady motion of a suspended sphere (Bassett, 1888) to obtain criteria for acceptable particle sizes in air as a function of the maximum turbulence frequencies expected. (For oil flow in a converging channel, McEligot and Eckelmann [2006] found maximum significant frequencies to be less than unity in non-dimensional wall coordinates, $f v / u_{\tau}{ }^{2}$.) Particle concentration was assumed to be low, and Stokes drag law was employed. As an air flow, only the acceleration force and viscous resistance were considered in the equation of motion; for liquid flows the pressure gradient and added mass terms should also be included. Particle response is presented as a function of a characteristic frequency defined as

$$
C=\frac{18 \mu}{\rho_{p} d_{p}^{2}} .
$$

Seeding particles, which are apparently satisfactory for liquid flows, are listed in Melling Table 4; he also discussed generation of scattering particles, primarily for air flows.

For their liquid flow, Soranna et al. (2006) cite Sridhar and Katz (1995) in indicating that slip between their particles and fluid would have been less than $0.2 \%$.

Developments concerning unsteady dynamic forces on a particle were reviewed by Mei (1996). Mei derived particle frequency response and energy transfer functions for solid particles and contaminated microbubbles in gas or liquid flows. A simple method for estimating a cut-off frequency (or cut-off size) was developed in turn. The cut-off frequencies are defined as 50 or $200 \%$ response in terms of the defined energy transfer function $|\mathbf{H}\{\omega\}|^{2}$, where $\omega$ is the frequency. This function depends on a Stokes number called

$\varepsilon=\sqrt{\frac{\omega a^{2}}{2 v}}(a$ is particle radius $)$

and the particle-to-fluid density ratio 
$\rho=\frac{\rho_{p}}{\rho_{f}}$

For a neutrally buoyant particle, $\rho=1$ and then $|H\{\omega\}|^{2}=|H\{\varepsilon\}|^{2}=1$, implying perfect particle response. On the other hand, for large $\rho$ and large $\varepsilon$, a low-pass filtering behavior of heavy particles is implied, giving practically negligible frequency response. The behavior of the function $|H\{\varepsilon\}|^{2}$ (Figure 1 by Mei, 1996) indicates that the range $0.56<\rho<1.62$ should provide very good particle response; outside this range, interpolation formulae are given to estimate the cut-off frequency (Mei, 1996 Eqns. 24b and 25).

The particles in the MIR VHTR experiment are silver-coated hollow glass spheres with a nominal mean particle diameter of about $14 \mu \mathrm{m}$ with $90 \%$ less than $21 \mu \mathrm{m}$, and their density is estimated as 1.65 $\mathrm{g} / \mathrm{cc}$ The density ratio is then about 2. Using the approximations of Mei with kinematic viscosity of about $14 \mathrm{cS}$ for the oil and $\mathrm{a}=0.5$, and $\mathrm{d}_{\mathrm{p}}=10.5 \mu \mathrm{m}$, one finds the estimated cutoff frequency to be above 40 $\mathrm{kHz}$. The frequency of shedding from the posts in the MIR model is estimated to be of the order of $1 \mathrm{~Hz}$. For order-of-magnitude estimates of the maximum turbulent frequencies near the surfaces, one could estimate the level of the wall shear stress and then apply the non-dimensional spectra measured by McEligot and Eckelmann (2006), also in an oil flow.

An alternate approach to examining the question of particle motion is to put it in terms of settling velocity (or terminal velocity due to gravity). The terminal velocity for a small particle falling through a stagnant fluid due to gravitational forces can be estimated via a force balance for steady motion, shown here as

$$
\frac{d V}{d t}=0=\frac{4}{3} \pi a^{3}\left(\rho_{p}-\rho_{f}\right) g-6 \pi \mu a \varphi V
$$

(Mei, 1996). The first term is the difference between the gravitational force and buoyancy while the second represents drag on the particle. The quantity, $\varphi$, is a correction factor relative to Stokes drag; it approaches unity as the particle Reynolds number approaches zero. This relation can be rearranged to yield the settling velocity as

$$
V \approx \frac{2 a^{2} g}{9 v \varphi}\left(\frac{\rho_{p}}{\rho_{f}}-1\right) .
$$

For our particles of about $1.7 \mathrm{~g} / \mathrm{cc}$ and radii of 10 microns in our oil, this estimate gives about $0.02 \mathrm{~mm} / \mathrm{s}$ or less and $\mathrm{Re}_{\mathrm{p}}$ about $3 \times 10^{-5}$. The lowest flow velocity we might encounter would probably be about $1 \mathrm{~cm} / \mathrm{s}$. Thus, in this sense, we would expect the particles to follow the flow adequately for the PIV.

\section{Measurements}

Prasad et al. (1992) experimentally examined resolution of an autocorrelation approach using a centroiding technique for locating the correlation peak. When particle image diameter is small relative to pixel size, mean-bias error becomes significant due to finite numerical resolution of the correlation function. In the limit as the image becomes much smaller than the pixel resolution, the result will be the same irrespective of where the image is deposited on a given pixel. The maximum possible bias error for 
the location of a particle image equals one-half the pixel spacing $\left(\mathrm{d}_{\text {pixel }}\right)$ with the bias being towards the center of the pixel. The bias in the location of an individual particle image is not of critical importance in PIV since each vector calculation includes several images. However, the bias in locating the centroid of the signal peak is still present. The authors show that this bias error decreases as $d_{\tau} / d_{\text {pixel }}$ increases. A simulation showed sizable maximum errors for $\mathrm{d}_{\tau} / \mathrm{d}_{\text {pixel }}$ less than 2 . Experiments with $\mathrm{d}_{\tau} / \mathrm{d}_{\text {pixel }}$ of 1.9 and 3.8 with windows of $32 \times 32$ and $64 \times 64$, respectively, gave maximum bias errors of about 0.16 pixels.

Ullum et al. (1998) employed a Dantec processing system to examine uncertainties in PIV measurements of grid turbulence in a low-speed wind tunnel. They concluded that their 3,000 samples (vector maps) at a $5 \mathrm{~Hz}$ sampling rate were effectively and statistically independent. By processing limited numbers of samples (e.g., 5, 50, 500 samples at a time), they found that the variations of the mean statistics fell within theoretical estimates of the effects of sample size. Consequently, if uncertainties within $1 \%$ are desired with turbulence intensity of $10 \%, 100$ vector maps would be required for the mean velocity but 20,000 vector maps for a component of the Reynolds normal stress.

Luff et al. (1999) conducted a computational and experimental study to investigate the typical experimental uncertainties that could be expected in the use of PIV systems to measure vorticity (which is calculated via measured velocity gradients or their equivalents). They showed that average uncertainties up to $37 \%$ and peak uncertainties of the order of $550 \%$ could result from the direct numerical processing using autocorrelation techniques. Cross-correlated results were not as demanding. With appropriate processing, average uncertainties could be reduced to about $4 \%$ and peak uncertainties were on the order of $20 \%$. Various approaches to improving the situation are provided in the Luff et al. (1999) article.

Forliti, Strykowski, and Debatin (2000) examined bias and precision uncertainties of various peak-finding algorithms with TSI software. They found that bias uncertainties exist for all algorithms studied, and these uncertainties tend to affect the precision uncertainty. The bias uncertainty was found to depend functionally on the displacement gradients present in the interrogation region. Forliti and Strykowski (2005) applied these results to a wind tunnel experiment where they estimated that using a minimum of 500 velocity fields for statistics led to precision uncertainties in mean and fluctuating velocities of, nominally, $2 \%$ of the inlet velocity. Instantaneous velocity measurements were believed to have bias uncertainties of the order of $2 \%$ of the inlet velocity as well. Convergence was shown for a point in a highly turbulent region of a recirculation bubble; at this location the mean displacement of about 0.3 pixels had a precision uncertainty of about \pm 0.1 pixels.

In a water tunnel experiment on vortex shedding in a cylinder wake, Kim et al. (2004) found the reproducibility of the instantaneous local velocities to be within $3.4 \%$ of the free stream velocity $(72.4$ $\mathrm{mm} / \mathrm{s})$.

In measurements for flow around an operating rotor blade in a refractive-index-matched flow, Soranna, Chow, Uzol, and Katz (2006) employed a 2-D PIV system using in-house-developed software and procedures. With $32 \times 32$-pixel interrogation windows and $50 \%$ overlap, they estimated the uncertainty in mean displacement to be about 0.3 pixels, provided the window contains at least 5 to 10 particle pairs. For typical displacements of 20 pixels, the resulting uncertainty in instantaneous velocity was about $1.5 \%$. For their particles, slip due to density differences was estimated to cause errors of less than $0.2 \%$. Soranna et al. did not discriminate between bias and precision uncertainties.

Roth, Mascenik, and Katz (1999) used a PIV system to measure flow structure and turbulence within a ship bow wave in a tow tank at the David Taylor Model Basin. They describe several approaches to address image enhancement and their treatment for displacements in regions of high-velocity gradients. Convergence was also studied and reported. Velocities are computed using in-house-developed correlation software with an interrogation window size of $12.5 \mathrm{~mm} \times 12.5 \mathrm{~mm}(64 \times 64$ pixels $)$ and a distance of $3.1 \mathrm{~mm}$ (16 pixels) between adjacent windows for $75 \%$ overlap. The estimated uncertainty 
level was about 0.4 pixels. For a characteristic displacement of 9.64 pixels, the velocity uncertainty was about $4 \%$ and the vorticity uncertainty was about $27 \%$. They concluded that 92 realizations were sufficient for convergence of mean velocities (to some unspecified level) but not for Reynolds stress measurements.

Roth and Katz (2001) examined methods of improving the efficiency and accuracy of data analyses for PIV software. Accuracy can be improved by image enhancement, resolving velocities in high-gradient regions and eliminating peak locking. These approaches can be beneficial when employing in-house software but may not be applicable when one uses commercial systems.

\section{International PIV Challenges}

A useful series of studies and consequent reports is provided by the International PIV Challenges (Stanislas et al., 2003, 2005). In addition to the published journal summaries, the databases are available on their Internet website (www.pivchallenge.org). As noted by PIV Challenge authors, one of the benefits of the website is to help newcomers to PIV to check whether they are correctly handling the software they use.

The first challenge examined up to nine sets of single images, both synthetic and real. The aim was to undertake a detailed and quantitative comparison of the ability of different evaluation algorithms and software to analyze single exposed PIV and particle-tracking velocimeter (PTV) images. The overall agreement of the up-to-date PIV algorithms was deemed to be fairly good, even in regions where difficulties were expected. Some general conclusions were as follows:

- In each case, at least three teams came to agreement in the range of 0.1 to 0.2 pixels over most of the field.

- Most advanced PIV algorithms do not show strong peak locking (Westerweel, 2000b).

- One must be very careful about the definition of the location of the measured vectors, e.g., do we have an averaged vector over the area inside each $16 \times 16$ square, or is the vector at the center of the window or somewhere in between?

Fifteen teams exercised their PIV and PTV algorithms on three sets of turbulent time-series data in the second challenge. Two sets were from actual experiments and the third was based on synthetic images generated from direct numerical simulations (DNS) of turbulent channel flow. Global agreement between the different methods was considered to be good. One key conclusion was that synthetic images were not fully representative of real images. For Case A, an experiment with a submerged turbulent water jet, the overall agreement of the results for axial displacements was good. The remarkable differences were for radial displacements in the region where there was no turbulence, and displacements were uniform and small (well below 1 pixel). Values of the rms displacement error were reported at 0.05 to 0.18 pixels, while radial displacements in the outer region were of the order of 0.10 to 0.15 pixels.

The aim of Case B was to assess the performance of PIV/PTV algorithms on a synthetic turbulent flow with strong velocity gradients. It employed 100 velocity maps from the DNS data base. For $\mathrm{y}^{+}>40$ ( $y>200$ pixels from the wall), mean velocity agreement was within 0.02 pixels. Bias was evaluated as being of the order of 0.02 pixels except near the wall. For the turbulent fluctuations ( $r m s \boldsymbol{u}$ and $\boldsymbol{v}$ ), the rms differences between measured and DNS values were of the order of 0.1 to 0.2 pixels away from the wall. Conceptually, these are random uncertainties propagated from larger instantaneous random uncertainties through the averaging process. Instantaneous comparisons between calculated and DNS values were as good as 0.2 pixels away from the wall. If these are random uncertainties and the realizations are independent, 100 realizations would reduce the uncertainties in the mean values by a factor of 10 , giving 
the order of the bias uncertainty presented in their Figure 14. Case C examined time-resolved PIV systems with a water flow experiment over an airfoil section.

The third challenge was held in Pasadena, California, during September 19-20, 2005, but the results are not yet published. However, informal minutes are available (C. Surmann, LaVision, e-mail, 11 July 2006). Five cases were examined, three with synthetic images and two with real images:

- Images to check spatial resolution

- Time-resolved PIV in a channel flow

- $\quad$ Time-resolved PIV in a jet flow

- Stereo PIV accuracy assessment

- Stereo PIV in a pipe flow.

Some preliminary conclusions were as follows:

- Multigrid, multipass with image deformation is a must

- PTV does not improve spatial resolution but brings high-frequency noise

- Window deformation algorithms improve stereo PIV

- Accuracy of stereo PIV is equivalent on the three components and is limited by the accuracy of the PIV algorithm

- $\quad$ Adequate pre- and post-processing are mandatory.

\section{MIR LOWER PLENUM EXPERIMENT}

The MIR experiment will simulate flow features of the paths of jets as they mix in flowing through the array of posts in a lower plenum en route to its single exit duct.

\section{INL MIR Flow System}

Unheated MIR experiments are first steps when the geometry is complicated (Stoots et al., 2001). Useful optical flow measurements in this realistic configuration would be impractical without refractive index matching.

The benefit of the MIR technique is that it permits optical measurements to determine flow characteristics in passages and around objects to be obtained without locating a disturbing transducer in the flow field and without distortion of the optical paths. With a transparent model of a different refractive index than the working fluid, the optical rays can be refracted in such a manner that measurements are either impossible (e.g., cannot see the desired location) or require extensive, difficult calibrations. Thompson, Bouchery, and Lowney (1995) demonstrated this situation conceptually when laser Doppler velocimetry (LDV) is applied to a rod bundle; with refractive index matching, the measurement and determination are relatively straight forward, while without refractive index matching, the beams may not cross to form the measurement control volume at the desired location, if they cross at all. The MIR technique is not new; Corino and Brodkey (1969) employed it to measure turbulence structure in a circular tube. Recent applications of the technique include, but are not limited to, those of Durst, Jovanovic, and Sender (1993), Parker and Merati (1996), Cui and Adrian (1997), and Becker et al. (2002).

The innovative advantage of the INL system is its large size, leading to improved spatial and temporal resolution compared to others. To date, most other experiments with index matching have been small, with characteristic lengths of $5 \mathrm{~cm}$ or less. In contrast, the INL MIR test section has a cross section of 
about $60 \times 60 \mathrm{~cm}$ and is about two meters long, allowing the use of models of substantial size. Because the system volume is over 3,000 gallons, a light mineral oil was selected as the working fluid due to environmental and safety considerations; its refractive index matches that of some quartz. With the fluid temperature controlled, the quartz model can barely be seen at some wave lengths in the visible spectrum; indices are typically matched for the wave length of the laser employed. The design flow rate can give Reynolds numbers up to about $10^{5}$ based on the cross section of the test section. The refractive index of the fluid is maintained at the desired value by a parallel temperature control system, which maintains a constant temperature in the test section within $\pm 0.05^{\circ} \mathrm{C}$. In measurements in an experiment on transition induced by a square rib, meaningful velocity and turbulence data were obtained as close to the surface as $\mathrm{y}^{+}=\left(\mathrm{y} \mathrm{u}_{\tau} / v\right) \approx 0.1$ and less (Becker et al., 2002).

Because the refractive index varies with temperature, it is necessary to conduct MIR experiments with a constant, uniform temperature in both the internal flow model and the external flow around it in the main test section. To maintain the flow through the model at the required temperature, an auxiliary flow loop temperature control system (Figure 36 in Condie et al., 2005) has been developed to be comparable in operation to the successful temperature control system for the main flow loop. It includes a heat exchanger to remove the energy introduced by the model loop pump, an electrical heater and its control for fine adjustment and a pump and flowmeter unit. The temperatures of both the main loop and the model loop are controlled through a LabView program.

Durst, Keck and Kleine (1979) have suggested that the refractive index must be maintained within \pm 0.0001 for high-quality LDV measurements. For the mineral oil in the INL MIR system, this criterion translates to the requirement that the oil temperature must be held steady and uniform to within about $0.3^{\circ} \mathrm{C}$; in general, we aim at a limit of about $0.1{ }^{\circ} \mathrm{C}$. Experience has shown that, after the initial transients, it is possible to maintain the test section temperature as close as possible to $\pm 0.02^{\circ} \mathrm{C}$ and the model temperature range to $\pm 0.05^{\circ} \mathrm{C}$, which is well within the desired limitations. It has been found that at the maximum model flow rate and a room temperature of about $32^{\circ} \mathrm{C}\left(90^{\circ} \mathrm{F}\right)$, the system could maintain the desired oil temperature of approximately $24^{\circ} \mathrm{C}\left(75^{\circ} \mathrm{F}\right)$ to within about $\pm 0.08^{\circ} \mathrm{C}$.

This MIR flow system (Stoots et al., 2001) has been used for fundamental and applied measurements in a variety of recent research programs:

- Transition in boundary layers with the Lehrstuhl für Strömungsmechanik of Uni. Erlangen, Germany (Becker et al., 2002)

- Flow phenomena in SNF canisters for a Department of Energy Office of Environmental Management (DOE-EM) Science project (McCreery et al., 2002)

- Flow fields around buildings for assessment of Bechtel Research and Development (R\&D) computer simulations for fate and transport of biological agents (McCreery et al., 2001)

- Effects of realistic surface roughness on turbomachinery flows with the University of Idaho for the Air Force Office of Scientific Research (Budwig et al., 2001)

- Complex flows relating to advanced gas-cooled nuclear reactors for the DOE NERI program (McCreery et al., 2003)

- Complex flows relating to supercritical water reactors for the DOE US/Korea I-NERI program (McEligot et al., 2005)

- Synthetic jet actuators by the University of Wyoming for the Air Force Office of Scientific Research (Shuster et al., 2005).

Flow field and turbulence measurements are primarily conducted by optical techniques: PIV and LDV. Both have advantages, and we consider them to be complementary approaches. Visualization of 
mean flows and instantaneous measurement of three velocity components are available via the PIV system. For flow visualization and for measuring mixing, the PTV may be useful. LDV gives timeresolved measurements. With the PIV, one needs many realizations to deduce means and the higher-order moments. We apply LDV a point at a time; 3-D measurements are obtained by traversing (taking profiles) in three directions in turbulent flows that are steady in the mean. Provided the application can be considered to be at least quasi-steady (residence times quicker than other response times), useful data for assessment can be obtained from such steady flows. Typical results include time-resolved, pointwise distributions of the mean velocities, $\mathrm{U}, \mathrm{V}, \mathrm{W}$, and their Reynolds stress components. With LDV, the time series are also available for spectral and wavelet analyses to investigate potential shedding of eddies from blunt bodies, such as support posts in a lower plenum.

The LDV system is a two-component, TSI fiberoptic-based laser Doppler velocimeter. Transmitting (and receiving) optics are provided by a Model 9832 two-component fiber optic probe with a lens of $350 \mathrm{~mm}$ focal distance. Included in the probe is a Model 9832-XX-Lens collimating lens option to reduce the measuring control volume; the diameter is estimated to be about $60 \mu \mathrm{m}$ in the MIR mineral oil. Signal analysis and data processing are accomplished via a Model S65-2 two-component signal analysis system including a two-channel IFA 655 digital burst correlator and FIND-W (low INformation DisplayWindows-based) software. For acquiring additional signals such as flow rate, temperature, and hot-film measurements simultaneously, a TSI Model DL4 four-channel Datalink multichannel interface is available. Data acquisition is controlled via the FIND-W software for the LDV system. Desired traversing locations plus measuring parameters are specified by the operator and then the sequence is initiated or positions may be adjusted via manual control. Data reduction to determine mean quantities and/or spectra is accomplished later, using other FIND-W subprograms.

Further details of the MIR flow system are provided in a technical paper by Stoots et al. (2001) and on an INL website (http://www.inel.gov/env-energyscience/physics/mir/). Some estimated experimental uncertainties are summarized by Becker et al. (2002).

\section{INL MIR PIV System}

The PIV system from LaVision, Inc. (Ypsilanti, Mich.) can be used for two-component or threecomponent instantaneous and mean-velocity component measurements in a two-dimensional laser light sheet. A vertical light sheet is provided by dual-cavity-pulsed Nd:YAG laser from Big Sky Laser Technologies (Bozeman, Mont. 59715) with maximum power of $120 \mathrm{~mJ} /$ pulse at $532 \mathrm{~nm}$ at a maximum pulse rate of $15 \mathrm{~Hz}$ and adjustable light sheet optics. Minimum light sheet thickness is about $1 \mathrm{~mm}$. The lasers and optics are mounted on a two-directional traversing system under the MIR test section so that the light sheet can be positioned by a stepping motor to within $\pm 0.005 \mathrm{~mm}$ in the cross-stream direction; its position is measured to within about $0.005 \mathrm{~mm}$ with a linear encoder and digital readout. One or two cameras view the light sheet from mountings on the LDV traversing mechanism, giving 2-D and 3-D capability, respectively. The cameras are the LaVision Imager Pro Plus $2 \mathrm{M}$ model with $1648 \times 1214$ pixel resolution and 14-bit dynamic range with two-stage Peltier cooling and the possibility of better than 100ns interframe time. Lenses with $28 \mathrm{~mm}, 50 \mathrm{~mm}$, and $105 \mathrm{~mm}$ focal lengths are available for large and small fields of view. The system computer is a dual processor PC with $\mathrm{P} 4$ processors. These processors have speeds of $2.8 \mathrm{GHz}$ and storage of $1 \mathrm{~GB}$ RAM, $120 \mathrm{~GB}$, and $300 \mathrm{~GB}$ internal hard drives, and a 135 Serial ATA Mobile Rack with SATAII 150 Serial ATA Controller that supports 500 GB removable hard drives, read/write DVD, 1.44 MB floppy drive, and an external Sony AIT-1 tape drive for 91 GB tapes. A Windows XP operating system is employed. Software has both PIV and PTV algorithms and provides instantaneous and mean-velocity components, turbulence statistics and velocity gradients across the image from the three-dimensional light sheet. 
The camera view is oriented to give an image of about 1,600 pixels wide (horizontal) and 1,200 pixels tall (vertical). The pixel size is $7.4 \times 7.4$ microns, and the pixels in the image appear to be square in the enlarged images.

According to Anderson (Anderson, e-mail, 6 July 2006), the calculated velocity vector is placed at the center of the interrogation window based on the first exposure, and the interrogation window for the second exposure is shifted by the local velocity vector. The vector value that is reported represents a average displacement of all particle images in that interrogation window.

Table 1 displays typical settings used in the MIR 3D-PIV experiments.

Table 1. Typical INL MIR PIV experiment settings.

\begin{tabular}{|l|l|}
\hline \multicolumn{1}{|c|}{ PIV Setting } & \multicolumn{1}{c|}{ Setting Used } \\
\hline Camera recording mode & 3 -D cross correlation \\
\hline PTU trigger & Internal \\
\hline Internal trigger rate $(\mathrm{Hz})$ & $2-15$ \\
\hline Recording frequency $(\mathrm{Hz})$ & $2-15$ \\
\hline Laser frequency $(\mathrm{Hz})$ & $2-15$ \\
\hline Delay (ms) & 4 \\
\hline Laser control [dt $(\mu \mathrm{s})]$ & $10-300$ \\
\hline Laser power $(\%)$ & $20-65$ \\
\hline Laser Q-switch at maximum power (laser1/laser2) & $175 / 175$ \\
\hline Laser Q-switch at minimum power (laser1/laser2) & $370 / 385$ \\
\hline Image acquisition method and number of images & $\begin{array}{l}\text { Standard: 500-750 } \\
\text { RAM: } 170\end{array}$ \\
\hline Camera operating mode & $\begin{array}{l}\text { Calibration: single frame/single exposure } \\
\text { Recording: 3-D cross correlation }\end{array}$ \\
\hline Binning & $\begin{array}{l}\text { x and y off } \\
\text { Only software }\end{array}$ \\
\hline Calibration target & LaVision 3D-PIV Type 20 \\
\hline Batch processing setup & $\begin{array}{l}\text { PIV } \\
\text { No image processing } \\
32 \times 32 \text { pixel, two passes with 50\% overlap } \\
\text { Vector peak ration Q }<1.3 \\
\text { Median filter (strongly remove and iteratively } \\
\text { replace) }\end{array}$ \\
\hline
\end{tabular}

\section{Experiment and Model}

The flow in the lower plenum model can locally be considered to be a situation of multiple buoyant jets into a confined density-stratified crossflow, with obstructions. The hot flow from the coolant channels through the lower plenum to the core outlet duct encounters a very complicated geometry. Because the flow converges ultimately to a single outlet, the hot jets encounter different crossflow velocities depending on their locations relative to the outlet. The jets furthest from the outlet essentially exhaust into 
stagnant surroundings between the adjacent posts with the exception of the flow that they induce. On the other hand, the last row of jets before the outlet encounters crossflow from all the other jets. If a hot channel region is exhausted via one of the jets furthest from the outlet, there is concern that its impingement on the floor of the lower plenum may be too hot for the thickness of the insulation layer protecting the metallic core support plate below. The "hot streaking" issue pertains primarily to the entrance of the hot outlet duct to the turbomachinery. If a "hot channel" region exhausts through one of the last jets before the outlet duct, there is concern that it may not mix (and thereby cool) sufficiently before flowing along the metallic outlet duct.

The purpose of the fluid dynamics experiments is to develop benchmark databases for the assessment of CFD solutions of the momentum equations, scalar mixing, and turbulence models for typical prismatic VHTR plenum geometries in the limiting case of negligible buoyancy and constant fluid properties. As indicated in the section on Scaling studies in Condie et al. (2005), in normal, full-power operation of a typical VHTR conceptual design, buoyancy influences should be negligible in the lower plenum. The MIR experiment will simulate flow features of the paths of jets as they mix in flowing through the array of posts in a lower plenum en route to its single exit duct. General characteristics of the flow in the lower plenum have been deduced, and, these characteristics, desirable features of MIR experiments have been identified. Two conceptual model designs have evolved: one will model flow across an array of posts as near the outlet duct or in line with the duct centerline at the opposite side of the reactor, and the second partially simulates flow aligned between a row of support posts. The present experiment design addresses the first concept. Measurements will be obtained via PIV and/or by LDV.

The first experiment addresses flow in the region on the central plane away from the plenum outlet duct. Conceptually, there is no significant flow from the outer reflector (except some bypass flow between the prismatic blocks) and the dominant flow comes from the jets issuing from short coolant ducts at the corners of the hexagonal blocks (corner channels). The main plenum crossflow evolves from these jets in the active core and then passes below the central reflector, which lacks the corner coolant ducts (i.e., no additional jet inflow).

The model is scaled to the geometric dimensions of the NGNP Point Design (MacDonald et al., 2003) as an example of a typical prismatic VHTR. With a desired height-to-diameter ratio of about 7, optical constraints led to a post diameter, $\mathrm{D}_{\mathrm{p}}$, of $1.25 \mathrm{in}$. $(31.8 \mathrm{~mm})$. The nominal dimensions are

$\left(\mathrm{p} / \mathrm{D}_{\mathrm{p}}\right)=1.7,\left(\mathrm{H}_{\text {Plenum }} / \mathrm{D}_{\mathrm{p}}\right)=6.85$ and $\left(\mathrm{D}_{\text {Jet }} / \mathrm{D}_{\mathrm{p}}\right)=0.7$.

The model consists of a row of full circular posts along its centerplane with half-posts on the two parallel walls to induce flow features somewhat comparable to those expected from the staggered adjacent rows of posts in the reactor design. The posts and half-posts form an equilateral triangular pattern as in supporting a core consisting of hexagonal blocks. Posts, side walls, and end walls are fabricated from quartz to match the refractive index of the working fluid. In addition to the circular posts, a wedge-shaped quartz element has been fabricated to block the channel by replacing a post; it simulates the plenum wall formed by a hexagonal block of the outer reflector and will be mounted at the upstream end.

For a typical VHTR lower plenum, the flow from the coolant channels is collected in short ducts at the corners of the hexagonal blocks (corner channels). The flows from the coolant channels in the core are fully-turbulent and have approximately the same bulk velocities. A design goal for the jet inlet ducts is to simulate this situation so that the jets formed will have flow features comparable to those in a typical prismatic VHTR. Accordingly, aims for the design include an approximately uniform velocity with a high turbulence level and no significant swirl at the entry to the jet inlet ducts (about four diameters from their exits into the plenum). 
For $\left(\mathrm{D}_{\text {Jet }} / \mathrm{D}_{\text {Post }}\right)=0.7$ and 1.25 -in. posts, the nominal diameter of the jet inlet ducts is $0.87 \mathrm{in}$. $(22.2 \mathrm{~mm})$. Up to four inlet jets will be accommodated with individual control and flow metering. The choice of four jet inlet ducts is consistent with the number near the centerplane in the NGNP Point Design with three rings of hexagonal blocks in the active core.

For the first experiment, maximum jet flow rates will be limited by the capacity of the existing pump for the model flow system, by the number of jets and by the flow resistance of the jet supply system. With a grid to induce turbulence at the duct entry, it is expected that a Reynolds number of 3,500 to 4,000 (based on diameter) will reliably give sustained turbulent flow. Currently, the maximum flow rates possible are estimated to give $\mathrm{Re}_{\mathrm{D}} \approx 12,000$ for each of four jets.

\section{Estimates of PIV Uncertainties}

In addition to the uncertainties in velocity and turbulence measurements by PIV algorithms, other experimental uncertainties must be estimated for proper assessment of proposed CFD codes. These include, but are not limited to, the geometry of the model, the properties of the working fluid, flow rates, and consequently Reynolds numbers.

\section{Velocity Uncertainties}

Because the basic measurement of a PIV system is an instantaneous velocity component deduced from the translation $\Delta \mathbf{x}_{\mathbf{p}}$ of a group of particles over a time interval $\Delta t$,

$$
V_{p}=\frac{\Delta x_{p}}{\Delta t}
$$

where the subscript $\mathbf{p}$ indicates a measurement in pixels of the recorded image, the basic percent uncertainty in the velocity can be represented as

$$
\left(\% U n c V_{p}\right)=\sqrt{\left(\% U n c \Delta x_{p}\right)^{2}+(\% U n c \Delta t)^{2}} .
$$

The propagation of such uncertainties into the mean statistics reported then depends on the extent to which the uncertainties are random (precision) or systematic (bias) and the manner of presentation of results. In some situations, fixed uncertainties can be removed from the presentation by normalizing the quantities involved. The averaging process for determining mean statistics will reduce the resulting percent uncertainties when they are random.

Tutorial slides from LaVision (2002; Anderson, electronic communication, 31 March 2006) provide estimates of the accuracy of vectors for calculations done with their standard FFT correlation. The slides are probably based on synthetic data with somewhat Gaussian-shaped particle images (Anderson, electronic communication, 21 April 2006). Bias and rms error likely correspond to the mean bias and rms errors as defined by Huang, Dabri, and Gharib (1997). With 20 particles in an interrogation area of $32 \times$ 32 pixels, bias and rms errors vary from about 0.025 to 0.1 pixels for displacements $\Delta \mathbf{x}$ of 1 to 10 pixels. With the IA $=16 \times 16$ pixels and 200 particles, these uncertainties fall in a range of about 0.1 to 0.2 pixels up to $\Delta \mathrm{x}$ of 7 pixels - and then they explode. By employing an adaptive multipass technique, first with 64 $\times 64$ pixels and then $32 \times 32$ pixels, bias and rms errors are reduced to about 0.03 pixels for up to 10 pixel displacements. They suggest that rms-error is a function of the following factors:

- Size of interrogation window

- $\quad$ Number of particles (best $>10$ ) 
- Local velocity gradient inside interrogation window

- Non-matching particles $\left(\mathbf{V}_{\mathbf{z}}\right)$ and all sorts of noise.

LaVision also suggests that rms-error (random uncertainty) is typically 0.05 to 0.1 pixels in real data.

LaVision (2002) notes that particle image diameter of less than 1 pixel can cause bias during vector calculation. They recommend sizes of at least 2 pixels via slight defocusing and/or better algorithms, such as window deformation. The problem apparently is related to peak locking (Westerweel, 2000b).

In an ideal evaluation of PIV results, which analyzed synthetic particles generated from a DNS database, Stanislas et al. (2005) found discrepancies from instantaneous displacements of about 0.5 pixels near the wall and 0.2 pixels further away; it is not clear whether these uncertainties were systematic or random. As an example, one PIV vendor recommends adjusting the timing so that the mean particle shift is about 5 pixels with a range from 0.1 pixel to $25 \%$ of the size of the integration window; this recommended mean particle shift would correspond to an uncertainty of about $10 \%$ near the wall (or $500 \%$ for the low end of the range). Multiple processing at different time intervals may be necessary to obtain desired accuracy in flows with wide ranges of velocities.

The processing algorithm for the LaVision PIV system gives uncertainties in displacements of about 0.05 pixels for synthetic images (Anderson, e-mail, 5 December 2005). For images of real experiments additional factors involved include calibration, focus, displacement of particles, particle seeding, spatial gradients within each interrogation spot, image contrast, operator settings, etc. Therefore, the value of 0.05 pixels probably can be considered to be about the best possible under ideal circumstances.

For the LaVision PIV system, the random uncertainty in the timing is estimated to be about $1 \mathrm{~ns}$ (Anderson, e-mail, 23 March 2006). For the typical pulse separation of $100 \mu$ s or more used for the MIR $\mathrm{PIV}$, the uncertainty would be $\leq 0.001 \%$ (McIlroy, e-mail, 10 March 2006) and therefore negligible compared to the displacement uncertainty.

In three-dimensional operation, the laser sheet thickness may be determined from measuring a correlation peak once a self-calibration procedure is completed. For the current operations, this thickness is estimated to be $2 \pm 0.2 \mathrm{~mm}$ (McIlroy, e-mail, 29 August 2006).

To convert the basic PIV observation to actual velocities a scaling factor, $\mathbf{S}$, is employed relating the pixel dimensions to physical distance in the field of view with units of pixel $/ \mathrm{mm}$. This quantity can suffer from both systematic (or fixed) and random uncertainties. The physical distance can be provided from a calibration device, fabrication sketches with tolerances, as-built drawings and/or independent measurements. The connection to the pixel grid is obtained via the camera view for the same location(s). Conceptually, use of a calibration procedure with a calibration device can account for parallax in the camera views. Since several of these measurements are accomplished optically, they can be subject to refractive index variations, which can be either systematic or random, depending on the temporal and spatial scales involved.

In three-dimensional operation with two cameras, a LaVision 3-D Type 20 calibration target and related software are employed to relate the observed image dimensions in pixels to physical distances in the oil. From this determination a scale factor can be calculated in pixels $/ \mathrm{mm}$ or vice versa. Currently we do not have estimates of the uncertainties involved in this process.

The particles in the MIR VHTR experiment are silver-coated hollow glass spheres with a nominal mean particle diameter of about $14 \mu \mathrm{m}$ with $90 \%$ less than $21 \mu \mathrm{m}$ and their density is estimated as 1.65 $\mathrm{g} / \mathrm{cc}$ (McIlroy e-mail, 2 June 2006). The density ratio is then about 2. Using the approximations of Mei (1996) with kinematic viscosity of about $14 \mathrm{cS}$ for the oil and $\mathrm{a}=0.5 \mathrm{~d}_{\mathrm{p}}=10.5 \mu \mathrm{m}$, one finds the estimated cutoff frequency to be above $40 \mathrm{kHz}$. The frequency of shedding from the posts in the MIR 
model is estimated to be of the order of $1 \mathrm{~Hz}$ (McEligot notes, 30 March 2006). For order-of-magnitude estimates of the maximum turbulent frequencies near the surfaces, one could estimate the level of the wall shear stress and then apply the non-dimensional spectra measured by McEligot and Eckelmann (2006), also in an oil flow.

It is instructive to examine estimated experimental uncertainties, based on a realistic best case (such as the results of Stanislas et al. [2005]), relative to expected operating conditions in the MIR lower plenum experiment. The low flow rate conditions, with one jet operating at $\operatorname{Re}_{\text {Jet }}=4000$ and no imposed crossflow, provide a challenge. We consider arranging the camera view to cover the vertical extent of the model including about one diameter into the jet inlet ducts, giving a vertical view of about 10 in. $(250 \mathrm{~mm})$. If this height corresponds to the 1200-pixel orientation of the camera, the relation would be about $0.2 \mathrm{~mm} / \mathrm{pixel}(0.0083 \mathrm{in} / \mathrm{pixel})$. The highest velocity will be a bit higher than the jet inlet bulk velocity, say about $10 \mathrm{ft} / \mathrm{s}$ (three $\mathrm{m} / \mathrm{s}$ ). Recent practice in operation of the PIV system has been to select a time interval $\Delta \mathrm{t}$ of $100 \mu \mathrm{s}$ (McIlroy e-mail, 10 March 2006). With that time interval, the displacement $\Delta \mathrm{s}$ would be about 1.4 pixels. Stanislas et al. (2005) found typical displacement uncertainties of about 0.2 pixels for some cases away from walls in an ideal situation (and more near the wall). This uncertainty corresponds to a $14 \%$ uncertainty in the measured velocity in the MIR experiment. If it is a systematic uncertainty, then $14 \%$ would be about the best we could do. If it is a random uncertainty, the uncertainty in the mean value will be reduced depending on the number of independent realizations achieved.

\section{With}

$\sigma_{\text {Mean }} \approx \sigma_{i} \sqrt{N}$

200 realizations would reduce this random uncertainty to about $1 \%$. Since we are discussing the maximum velocity in the field, all other velocities would have greater percent uncertainties. This situation can be improved by increasing the time interval of each frame pair and by concentrating on a smaller physical field of view so the motion covers more pixels in the camera view. However, if $\Delta \mathbf{s}$ becomes too large, presumably it will become more difficult to correlate the particle motion and accuracy will suffer in that direction.

With the current MIR model and the 3D-PIV system, McIlroy and colleagues (2006) examined the effects of sample size for a typical set of measurements, using an approach comparable to that of Uzol and Camci (2001). They collected 3,000 images of a region below a jet inlet and evaluated the variations in mean statistics at seven locations as the number of samples was varied from 100 to 1,500, using a postprocessing code developed by D. Christensen of Utah State University. Mean velocities varied from about 9 to $0.7 \mathrm{~m} / \mathrm{s}$ for these seven positions and mean-squared fluctuations varied from about 0.05 to $4 \mathrm{~m}^{2} / \mathrm{s}^{2}$. About $10 \%$ of the vectors under the jets and at the bottom of the plenum in the slower region were labeled invalid by LaVision software, while in the middle of the plenum about $30 \%$ or more were invalid. The mean statistics calculated were based on the numbers of valid vectors at each point. Thus, for 1,000 samples there would have been about 700 to 900 individual realizations, depending on the location.

This approach evaluates effects of random uncertainties on the mean statistics for this particular situation (view and processing). Trends were generally as observed by Uzol and Camci. Preliminary conclusions were that, for this view and processing parameters, about 750 images should be collected to reduce the scatter in mean velocity statistics to between 0.4 and $10 \%$ for velocities greater than about $2 \mathrm{~m} / \mathrm{s}$.

For this study of sample size, the time interval $\Delta \mathrm{t}$ was $120 \mu$ s and the scaling factor was about 9.4 pixels $/ \mathrm{mm}$, so the estimate of random uncertainties would be about $0.3 \mathrm{~m} / \mathrm{s}$, using a displacement uncertainty of 0.3 pixels. Mean velocities varied from about $9 \mathrm{~m} / \mathrm{s}$ in the jet to $0.7 \mathrm{~m} / \mathrm{s}$ in the middle of the plenum. Corresponding instantaneous uncertainties would be about 3-40\%, respectively. For 1,000 
samples, these values would lead to predictions of uncertainties of about 0.1 and $2 \%$. The former is consistent with the scatter observed in the sampling study but the latter is about an order-of-magnitude less. Approximate levels of mean-square-fluctuations were 0.05 and $1.5 \mathrm{~m}^{2} / \mathrm{s}$ for these two extremes (giving turbulence intensity over $100 \%$ in the second case). The resulting random uncertainties are then 0.003 and $0.02 \mathrm{~m}^{2} / \mathrm{s}^{2}$, respectively. Both of these values are less than the scatter observed in the data processing.

The trends predicted by this uncertainty analysis are consistent with the observations but, for the most part, the expected values are less than the scatter observed. Possible explanations are that the displacement uncertainty has been underestimated, that a periodic process such as eddy shedding is affecting the results and/or some other unknown reason(s). Preliminary conclusions from the comparison are (1) more samples should be collected than predicted by the uncertainty analysis, and (2) longer time intervals, $\Delta \mathrm{t}$, should be employed for the low-velocity regions.

\section{Positioning Uncertainties}

It does not do the CFD analyst much good to provide velocity statistics with small uncertainties if the locations are not known relative to the geometry.

The three-directional traversing mechanism was designed to use the INL LDV from TSI in the forward-scattering mode and to avoid relative motion between the test section and the optics. However, it is also employed to support the PIV cameras and can be used to measure model locations in conjunction with a PIV camera and its cursor. For vertical motion, platforms on each side of the test section are moved simultaneously under precise computer control to maintain alignment of the transmitting and receiving optics. The position is determined with an Accu-Rite linear encoder with digital readout with manufacturer's specifications of an accuracy of $\pm 0.0001 \mathrm{in}$. or about $2.5 \mu \mathrm{m}$. The digital readout displays in increments of $2 \mu \mathrm{m}$. Indicated variation in position while the system is warming is about $5 \mu \mathrm{m}$. Thus, the absolute uncertainty of a position is $5 \mu \mathrm{m}$, but for positioning during a continuous traverse the relative positioning uncertainty is less. The positional accuracy becomes important in deducing the wall shear stress and the wall location from fitting measurements for $\mathrm{y}^{+}<\sim 3$.

For two-dimensional PIV measurements with the light sheet parallel to a window and the single camera oriented for a perpendicular view, model positions can be determined with the PIV camera and the LDV traversing system as indicated above. McIlroy and Pink (2005) employed this technique to measure the positions of model rods and spacers relative to the traversing system in a comparable experiment. The cursor of the camera was set at the center of the view $(x=800$ pixels, $y=600$ pixels $)$ to avoid parallax and then the traversing system was moved (relative to a specified reference position) to measure the desired model position. It is estimated that the camera can be controlled so that the cursor is located at the position to be measured within \pm 1 pixel. In that experiment and camera position, 1 pixel corresponded to about $0.1 \mathrm{~mm}$. Locations are recorded to the level of accuracy of the LDV traversing system (i.e., about $0.002 \mathrm{~mm}$ ) and comparisons to known dimensions, such as rod diameters, agreed to within the fabrication tolerances (e.g., $0.05 \mathrm{~mm}$ ). Thus, it appears that the camera positioning procedure may yield determinations to better than the 1 pixel expected.

In MIR experiments with PIV measurements, the spanwise locations of the data are determined by the position of the vertical laser light sheet. The laser system is presently mounted below the test section on a PTV traverse designed by Prof. R. S. Brodkey and colleagues at Ohio State University as part of an earlier project (Crepeau et al., 1998). This traverse stage was aligned to within $\pm 0.002 \mathrm{in} .( \pm 0.041 \mathrm{~mm})$ of parallel with the rails of the test section traversing cage and to within $\pm 0.012 \mathrm{in} .( \pm 0.308 \mathrm{~mm})$ of level (Mcllroy and Pink, 2006). In the present experiment, the fundamental spanwise reference point was chosen as the vertical centerline of Jet 5 at the top of the model. For operation this reference has been 
translated to a secondary reference in view on the camera/LDV traverse cage above; to relate spanwise locations, the center of the light sheet may be positioned on this reference to within about \pm 0.002 in. $( \pm 0.041 \mathrm{~mm})$ and then traversed in $2 \mathrm{~mm}$ intervals. The traversing measurements then have uncertainties relative to spanwise position of about $2.1 \%$.

An additional spanwise uncertainty would be the effective location within the light sheet of the reported instantaneous velocity; presumably this quantity is related to the numbers and spatial distributions of the particles in the interrogation window and is unknown. One might guess a random uncertainty of about $1 / 10$ of the light sheet thickness in each image set; the random uncertainty in the mean position will be reduced via the multiple independent realizations $(\mathrm{N})$ sampled.

For measurements in the vertical plane of the light sheet (i.e., $\mathrm{x}$ and $\mathrm{y}$ ), the origin of the model coordinate system is taken at the upstream end of the model on the vertical centerplane where it intersects the top of the model flow passage (bottom of the upper quartz block). To determine locations within the image views, secondary reference positions have been established. For this purpose, holes have been bored vertically in two central quartz posts on their centerlines; pointed metal rods were installed in these holes to provide known reference points (within their fabrication tolerances). For views which do not include one of these reference positions, a reference is established at an intersection between the vertical centerplane and the upstream (or downstream) face of a central post where it meets the bottom of the upper quartz block or the top of the lower block. (Since the oil temperature is adjusted to match refractive indices at $532 \mathrm{~nm}$, it is possible to see quartz-oil interfaces in the camera view and at other wave lengths in the visible spectrum.)

The estimated uncertainty in absolute location of a secondary reference in model coordinates depends on the propagation of fabrication tolerances for post hole locations, post hole diameters, diameters of the ends of the posts and such. For the current experiment, it is estimated that a point in an image may be determined to within about $0.11 \mathrm{~mm}(0.0043 \mathrm{in}$.) by using the cursor and image magnification. The distance of $0.11 \mathrm{~mm}$ corresponds to one pixel when viewing with the $50 \mathrm{~mm}$ lens. Thus, a relative location between two points within the same image view may be determined to about $(2)^{1 / 2} \times 0.11 \approx 0.16 \mathrm{~mm}(0.006 \mathrm{in}$.). Since the cursor can be positioned to about $0.05 \mathrm{~mm}$ (approximately half the size of the center gap in the cursor crosshair), this estimate may be conservative. Measurements of post diameters by this technique routinely are within $0.11 \mathrm{~mm}$ of the design specifications, giving further confidence that this estimate is a reasonable value.

LaVision's DaVis software also has a measuring tool that is calibrated to model dimensions during the calibration procedure; it may be employed to determine distances between two points in a view but currently we do not have estimates of its experimental uncertainties.

During experiments the $\mathrm{x}, \mathrm{y}$ and $\mathrm{z}$ locations of the camera traverse and the $\mathrm{z}$ location of the laser light sheet are recorded for every file. This information may also be employed as a check to determine where a point in an image is located relative to the model origin.

\section{Estimates of Additional Contributing Measurement Uncertainties}

\section{Fluid Properties}

The key fluid properties in an MIR experiment are the fluid density, kinematic viscosity and - for optical calculations - the refractive index. These properties were measured at the University of Idaho by Orr, Thomson and Budwig (1998) and at INL by Glenn McCreery (McIlroy e-mail, 24 August 2006) as

Density $\rho=0.8449-0.0005883 \cdot T \pm 0.002(\mathrm{gm} / \mathrm{mL})$ 
Kinematic viscosity $v=269.39 \cdot T^{-0.9366} \pm 0.3(c S)$

Refractive index $n=1.463753-3.587 \times 10^{-5} \cdot(T-20) \pm 0.0003(c S)$

where $\mathrm{T}$ is measured in $\mathrm{C}^{\circ}$. During current experiments, operations routinely maintain $23.3^{\circ} \mathrm{C} \pm 0.02^{\circ} \mathrm{C}$ for the main test section (main flow) and $\pm 0.05^{\circ} \mathrm{C}$ for the model flow; these variations contribute to random uncertainties in measurement series. The related bias uncertainties are about $0.2,2$ and $0.02 \%$ for the calibrations of $\rho, v$ and $\mathbf{n}$, respectively. The random uncertainty in kinematic viscosity due to temperature fluctuations of $\pm 0.05^{\circ} \mathrm{C}$ would be $0.2 \%$.

\section{Geometry}

As-built measurements of model components have demonstrated that the resulting dimensions are within the tolerances specified in the fabrication sketches (Condie et al., 2005). For the key internal dimensions these tolerances are \pm 0.002 in. $( \pm 0.05 \mathrm{~mm})$ with the exception of the post heights which are \pm 0.005 in. $( \pm 0.13 \mathrm{~mm})$. The diameters of the jet inlet ducts are specified as $0.870 \pm 0.002 \mathrm{in}$. $(22.1 \pm 0.05 \mathrm{~mm})$ for bias uncertainties of about $0.2 \%$. (Section A-A in Figure A-5 of the fabrication sketch report has an error in this dimension.)

\section{Flow Rates}

In the first experiment, the flow is provided by one to four jets from the top of the model. The total flow rate is indicated by the flow through a turbine flow meter and a Coriolis flow meter in the model temperature control system as shown in Figure 16 of the report by Condie et al. (2005). However, the turbine flow meter is not calibrated and, in the past, has been found to be unreliable.

The flow rates to the jets are measured individually with four variable area flowmeters from Flowmetrics, Inc. (Chatsworth, Cal. 91311); they are designated as Part Number 2600VF8KVY-P08 where the letter $\mathrm{K}$ indicates a float fabricated from $316 \mathrm{SS}$. The manufacturer claims that they are calibrated for our approximate operating conditions to within $0.5 \%$ of reading, with an effective flow range of 2.5 to $60 \mathrm{gpm}$ of mineral oil. This uncertainty can be considered to be a bias uncertainty. Their tubes are sufficiently long that this claim may be reasonable. Typical minimum flow rates (for jet flows at $\mathrm{Re}_{\mathrm{Jet}} \approx 4000$ ) are about $15 \mathrm{gpm}$ so the float level can be expected to be near quarter-scale or above.

The principle of operation of the flowmeter is that the float configuration forms a sharp-edged annular orifice with the surrounding circular tube, with its differential pressure force balancing the effective weight of the float. A force balance in terms of the orifice loss coefficient (which is a function of level) can be derived to provide a relation between the velocity through the orifice and the liquid density; the geometric quantities are fixed at a given level. This relation can be rearranged to permit measurement with a fluid of one density after calibration with a fluid of another density. The loss coefficient is relatively independent of Reynolds number except at low flow rates (low Reynolds numbers) as in the example of Figure 5.3 by Sabersky et al. (1999). The manufacturer indicates that for this model the viscosity immunity ceiling is $46 \mathrm{cp}$ at a fluid specific gravity of 1.00 or, effectively, $14 \mathrm{cS}$. The conversion from one fluid to another will involve bias uncertainties due to uncertainties in the densities and, during experiment operation, random uncertainties from the effects of temperature variation on the density of the operating fluid.

Uncertainties in reading the flow rates from the meters would be random uncertainties of about onequarter to one-half division. The present meters have two scales. The glass tubes are scribed in millimeters from zero to the maximum of $600 \mathrm{~mm}$. A separate scale provides the calibration for an oil (at 
$100^{\circ} \mathrm{F}$ to give a kinematic viscosity of $14 \mathrm{cS}$ ) in intervals of $0.25 \mathrm{gpm}$ from 1.75 to $60 \mathrm{gpm}$. Current practice is to use the calibrated scale for setting and measuring the flow rate.

The specified oil density of the calibration is $0.83 \mathrm{~g} / \mathrm{mL}$, implying an uncertainty of $0.005 \mathrm{~g} / \mathrm{mL}$. This uncertainty converts to a bias uncertainty in measured flow rate of about $0.34 \%$, a fair fraction of the $0.5 \%$ claimed by the manufacturer. Our target oil temperature of $23.3^{\circ} \mathrm{C}$ gives an oil density of 0.8312 $\mathrm{g} / \mathrm{mL}$, which is well within the range of uncertainty of the calibration density.

Uncertainties in reproducing the specified flow rates of an experiment depend on the manner in which these flow rates are set. It is estimated that the flow rate settings can be repeated to within about 0.125 gpm (McIlroy, e-mail, 24 August 2006) for each jet.

The lowest flow rates of the experiment typically are $11.2 \mathrm{gpm}$ (float height about $12 \mathrm{~mm}$ ) for the first jet, which has a smaller cross section than the rest, and $16.7 \mathrm{gpm}$ (about $18 \mathrm{~mm}$ ) for the other three. Since the total volume flow rate is obtained by adding the flow rates from the individual jets operating, the absolute random uncertainty of the total is given by the absolute uncertainties of the number of jets operating as

$$
\delta Q_{\text {Total }} \approx \sqrt{N_{\text {Jets }}} \cdot \delta Q_{\text {Jet }}
$$

since their random uncertainties are the same.

At $11.2 \mathrm{gpm}$, the bias uncertainty would be $0.5 \%$ or about $0.06 \mathrm{gpm}$, and the random uncertainty can be taken as $0.125 \mathrm{gpm}$ or about $1 \%$. For the jets at $16.7 \mathrm{gpm}$, the bias uncertainty would be about 0.08 gpm and the random uncertainty would again be $0.125 \mathrm{gpm}$. As an example, if the first and third jets were operating, the bias uncertainty of the total would be

$$
\delta Q_{\text {Total }} \approx \sqrt{(0.056)^{2}+(0.084)^{2}} \approx 0.10 \mathrm{gpm}
$$

while for the random uncertainty it would be

$$
\delta Q_{\text {Total }} \approx \sqrt{2} \cdot 0.125 \approx 0.18 \mathrm{gpm}
$$

For this total flow rate of 27.9 gpm, the percent uncertainties would be about 0.4 and $0.6 \%$ for the bias and random uncertainties, respectively. At higher flow rates, the bias uncertainties would become larger than the random ones.

The percent uncertainties in the mass flow rates $(\dot{m}=\rho \cdot Q)$ are given via the percent uncertainties in the volume flow rates and the oil density which is given from the temperature measurements. In general, one can say

$$
\frac{\delta \dot{m}}{\dot{m}} \approx \sqrt{\left(\frac{\delta \rho}{\rho}\right)^{2}}+\left(\frac{\delta Q}{Q}\right)^{2} .
$$

\section{Reynolds Number}

The jet inlet Reynolds number is defined as $\operatorname{Re}_{J e t} \equiv \frac{V_{b} D_{J e t}}{v}$, which can be converted to 


$$
\operatorname{Re}_{J e t} \equiv \frac{4 \cdot \dot{m}_{\text {Jet }}}{\pi \cdot D_{J e t} \cdot \mu}=\frac{4 \cdot Q_{J e t}}{\pi \cdot D_{J e t} \cdot v}
$$

so the percent uncertainty in $\mathrm{Re}_{j}$ et can be estimated from the rms sum of the percent uncertainties of these variables. For a single jet, uncertainties can be estimated as

$$
\frac{\delta \mathrm{Re}_{\text {Jet }}}{\mathrm{Re}_{\text {Jet }}} \approx \sqrt{\left(\frac{\delta Q_{\text {Jet }}}{Q_{J e t}}\right)^{2}+\left(\frac{\delta D_{J e t}}{D_{J e t}}\right)^{2}+\left(\frac{\delta v}{v}\right)^{2}}
$$

At a flow rate of $11.2 \mathrm{gpm}$, the bias uncertainty would be

$$
\frac{\delta \mathrm{Re}_{\text {Jet }}}{\mathrm{Re}_{\text {Jet }}} \approx \sqrt{(0.0050)^{2}+(0.0023)^{2}+(0.0213)^{2}} \approx 0.022 \approx 2 \%(\text { bias })
$$

and the random uncertainty would be

$$
\frac{\delta \mathrm{Re}_{\text {Jet }}}{\operatorname{Re}_{\text {Jet }}} \approx \sqrt{(0.011)^{2}+(0.0023)^{2}} \approx 0.011 \approx 1 \%(\text { random })
$$

The bias uncertainty is dominated by the uncertainty in the calibration of $v$ and is relatively independent of the other two uncertainties. The percent random uncertainty will decrease as the flow rate increases.

\section{APPLICATION TO MIR LOWER PLENUM EXPERIMENT}

Taylor's (1997) "rule for stating uncertainties" is "experimental uncertainties should almost always be rounded to one significant figure."

The detailed approach to estimating the experimental uncertainties will depend on what results are desired and what information is available. In this section we provide an example of a case where physical velocities are known (e.g., from software output) or are approximately known (for design evaluation). For now, we concentrate on estimates away from a surface.

For this example, some 3D-PIV data for flows in the jet inlet ducts are considered (Mcllroy et al. 2006); however, the approximate values employed are not necessarily the same as in the experiment presented. Both bias (fixed) and precision (random) uncertainties are involved. Rood and Telionis (1991) discussed examples of both in a typical fluids engineering experiment but did not demonstrate their propagation. Kline (1985) shows the propagation for evaluation of density via the perfect gas

approximation $\rho=\frac{p}{R \cdot T}$ with negligible bias uncertainty for pressure but both bias and random uncertainties in the temperature. An rms combination is used separately until their resulting effects on the results are derived, and then these resulting bias and precision uncertainties are combined either by rms or linear approximations.

For our situation, we can say an instantaneous velocity is given by

$$
V=\frac{\Delta x}{\Delta t}=\frac{\Delta x_{\text {Pixel }}}{S \cdot \Delta t}
$$


where the scale factor

$$
S=\frac{\Delta x_{\text {Pixel }}}{\Delta x}
$$

with units of pixels/inch or pixels $/ \mathrm{mm}$. If the individual uncertainties are independent, the estimated percent (fractional [Taylor, 1997]) uncertainty in the instantaneous velocity would be

$$
\frac{\delta V}{V} \approx \sqrt{\left(\frac{\delta\left(\Delta x_{\text {Pixl }}\right)}{\Delta x_{\text {Pixel }}}\right)^{2}+\left(\frac{\delta S}{S}\right)^{2}+\left(\frac{\delta(\Delta t)}{\Delta t}\right)^{2}}
$$

i.e., for an individual realization. In estimating mean values, one can expect the first term to be primarily random and the second to be essentially a fixed (bias) uncertainty. For the LaVision PIV system employed in the INL MIR system, the jitter of the trigger pulses to the lasers and the jitter of the Q-switch are 1 ns combined (Anderson e-mail, 23 March 2006); this uncertainty would be random but inherently negligible (for $\Delta \mathrm{t}=100 \mu \mathrm{s}$ or more, the uncertainty is $0.001 \%$ or less).

From this discussion, we see that the fractional bias uncertainty simplifies to

$$
\frac{\delta V}{V} \approx \frac{\delta S}{S}
$$

with our present assumptions. (In a case with strong mean velocity gradients as near a wall, there may be additional contributions to the bias uncertainties.) To estimate a scale factor $\mathbf{S}$, we take some approximate values from a PIV photograph (for actual data reduction more precise calculations would be needed). The design distance from the upstream face of the duct for jet 3 to the downstream face for jet 4 would be $0.870+0.362+0.870=2.102$ in. from the fabrication sketches (Condie et al. 2005) with tolerances of about $\pm 0.002 \mathrm{in}$. on each of the three dimensions. The uncertainty would be $3.5 \times 0.002 \approx 0.0035 \mathrm{in}$. or $\frac{0.0035}{2.102} \approx 0.00167 \approx 0.2 \%$ (bias). Lacking specific information, we guess that this distance is about twothirds of the view width and that the figure width is the full 1,600 pixels or about 1,070 pixels. If that distance were actually measured with the camera, the uncertainty would probably be about one pixel for each of the two points, giving about 1.4 pixels for the difference, or about $0.25 \%$. The scale factor $\mathbf{S}$ is then $S \approx \frac{1070 \text { pixels }}{2.102 \text { in }} \approx 509$ pixels $/$ in. Its bias uncertainty can be estimated as

$$
\frac{\delta S}{S} \approx \sqrt{(0.00165)^{2}+(0.0025)^{2}} \approx .002995 \text { or } 3 \% \text { (bias) }
$$

For the maximum mean velocity in the jet inlet duct, we take $10 \mathrm{ft} / \mathrm{s}$ or $3 \mathrm{~m} / \mathrm{s}$ again $\left(\operatorname{Re}_{\text {Jet }} \approx 4000\right)$. With a time interval of $\Delta \mathrm{t}=150 \mathrm{~ms}$, this speed corresponds to $\Delta \mathrm{x}_{\mathrm{p}} \approx 4.9$ pixels. If the uncertainty $\delta\left(\Delta \mathrm{x}_{\mathrm{p}}\right)$ is about 0.3 pixels (random), this fractional uncertainty would be 0.061 , or about $6 \%$ for an individual realization. Based on the discussion above, the random uncertainty in the instantaneous velocity measurement would be

$$
\frac{\delta V}{V} \approx \sqrt{\left(\frac{\delta\left(\Delta x_{P i x l}\right)}{\Delta x_{\text {Pixel }}}\right)^{2}+\left(\frac{\delta(\Delta t)}{\Delta t}\right)^{2}} \approx \sqrt{(0.061)^{2}+\left(\frac{0.001}{150}\right)^{2}} \approx 0.061,
$$


demonstrating again that one can neglect the timing uncertainty.

The random uncertainty in the mean velocity would be

$$
\sigma_{r} \approx \frac{\sigma_{i}}{\sqrt{N}}(m / s)
$$

where $\mathrm{N}$ represents the number of independent realizations (Metzger 2002). A key question is the number of independent realizations. For the PIV data considered, usually 170 image sets were taken in 17 seconds, or about 0.1 seconds between sets of image pairs. If the flow were fully developed in the jet inlet duct, one could estimate a characteristic eddy-passing time from the bulk velocity $\mathrm{V}_{\mathrm{b}}$ and the tube diameter $\mathrm{D}$ as

$$
t_{c} \approx \frac{D_{r}}{V_{b}^{*}} .
$$

The bulk velocity at $\operatorname{Re}_{\mathrm{D}}=4,000$ is $\mathrm{V}_{\mathrm{b}} \approx 8.4 \mathrm{ft} / \mathrm{s}(2.6 \mathrm{~m} / \mathrm{s})$, giving $\mathrm{t}_{\mathrm{c}} \approx 0.0086$ seconds, or about 2,000 eddies in 17 seconds. Since the jet inlet ducts are short, the flow is a thinner boundary layer flow with a smaller characteristic thickness, giving a shorter characteristic time. Thus, if each image set provided a valid velocity vector at the location desired, the 170 sets would be independent. However, the PIV software does not necessarily obtain a valid vector for each image set. To calculate valid mean statistics, the LaVision software includes a parameter to set the minimum number of valid vectors (realizations) required at a node point; if the number is not attained, the mean statistics are not calculated there, and one sees holes in the results reported (Anderson e-mails, 13 March and 10 July 2006). Since the actual number of valid vectors is not reported, the most independent realizations one could claim would be this parameter: if the parameter is higher than the number of image sets, then, in this case, one could claim the full 170 sets for $\mathrm{N}$.

For convenience, we assume that the parameter is set for 100 valid vectors, so $\mathrm{N}=100$ at each node for which mean statistics are reported. For the values selected here, the random uncertainty of the mean velocity becomes

$$
\frac{\sigma V_{\text {Mean }}}{V_{\text {Mean }}} \approx \frac{\sigma V_{\text {Indiv }}}{V_{\text {Mean }} \sqrt{N}} \approx 0.0061 \approx 0.6 \% \text {. }
$$

For the uncertainty in the mean velocity at this location, one could report

$$
\left.\frac{\delta V_{\text {Mean }}}{V_{\text {Mean }}} \approx 0.6 \%(\text { precision }) \text { and } 0.3 \% \text { (bias }\right)
$$

Or, with rms combination,

$$
\frac{\delta V_{\text {Mean }}}{V_{\text {Mean }}} \approx 0.7 \%(\text { total }) \text {. }
$$

Metzger (2002) notes that, in evaluating the uncertainty of a fluctuation about a mean, the bias uncertainty cancels. Therefore, the experimental uncertainty of an rms velocity fluctuation (e.g., u'), Reynolds normal stress or such will only involve the random (precision) uncertainties. The resulting uncertainty in the root-mean-square value of the $\mathbf{u}$ ' fluctuation is given by 


$$
\sigma_{u^{\prime}} \approx \frac{1}{2} \frac{1}{\sqrt{N / 2}} \sigma_{u}
$$

where $\sigma_{\mathbf{u}}$ is the random uncertainty in an individual realization of the fluctuation $\mathbf{u}$. Because (due to the averaging process) the random uncertainty in the mean value $\mathbf{U}$ is considerably smaller than the random uncertainty of the instantaneous velocity, $\sigma_{u}$ is approximately the same as the random uncertainty of the instantaneous velocity ( $\sigma_{\mathbf{i}}$ above). Appropriate units of $\sigma_{\mathbf{u}}$ and $\sigma_{\mathbf{u}^{\prime}}$ are $\mathrm{m} / \mathrm{s}, \mathrm{ft} / \mathrm{s}$ or pixels $/ \mathrm{s}$, depending on the approach.

For the values of the example above, $\sigma_{u} \approx \sigma_{i}$ is about $6 \%$ of the $10 \mathrm{ft} / \mathrm{s}$ or $0.612 \mathrm{ft} / \mathrm{s}(0.187 \mathrm{~m} / \mathrm{s})$. Then $\sigma_{\mathbf{i}}\left(\right.$ or $\left.\sigma_{\mathbf{v}^{\prime}}\right)$ will be given by

$$
\sigma_{u^{\prime}} \approx \frac{1}{2} \frac{1}{\sqrt{100 / 2}}(0.612) \approx 0.0433(\mathrm{ft} / \mathrm{s}) \approx 0.013(\mathrm{~m} / \mathrm{s}) \approx 1(\mathrm{~cm} / \mathrm{s}) \text {. }
$$

The percent uncertainties can be calculated from the pointwise values of $\mathbf{u}^{\prime}$ (in $\mathrm{cm} / \mathrm{s}$ or such) produced by the PIV software.

Johnson (2006) developed an Excel spreadsheet and associated figures to present the distribution of estimated experimental uncertainties in instantaneous velocity results from the PIV system. Either random or bias uncertainties could be estimated if the displacement uncertainty dominated the result. A uniform distribution of displacement uncertainty was assumed.

Velocity results from the DaVis software of LaVision were exported to TecPlot 360 software so that any desired plane of data could be extracted. This plane was then transformed to a file for spreadsheet use. For each position, the following sequence of operations was accomplished:

1. The time interval, $\Delta \mathrm{t}$, of the image sets was specified.

2. Physical displacements in $\mathrm{mm}$ were calculated from the pointwise velocities.

3. The scale factor $\mathrm{S}$ was specified. This quantity was taken from the results of the calibration process for the 3D-PIV system.

4. Displacements were calculated in terms of pixels by applying the scale factor to the physical displacements.

5. A uniform displacement uncertainty was specified in terms of pixels. This quantity was estimated from consideration of the experience with the INL system and results in the literature. Either a random uncertainty or a bias uncertainty could be specified, giving random or bias estimates accordingly. Typically, a value of about 0.3 pixels was employed; this value is believed to be a conservative estimate. The estimated uncertainty in the time interval $\Delta \mathrm{t}$ was about one nanosecond, or typically less than $0.001 \%$, and, therefore, could be neglected.

6. Local fractional uncertainties were estimated by dividing this uniform displacement uncertainty by the local velocity. Multiplication by 100 would give percent uncertainties.

7. At each calculation step the pertinent distribution was presented as a descriptive contour plot in the plane of the observations.

Results of the spreadsheet calculation are estimates of fractional uncertainties for instantaneous velocity measurements. In general, the bias uncertainty will not vary with the number of samples, so, if 
the displacement uncertainty represents a bias uncertainty, the estimates for uncertainties in the instantaneous velocities and the time-mean velocities will be the same.

The random uncertainties in mean velocities and other mean statistics are reduced as the number of independent realizations, $\mathrm{N}$, is increased, in accordance with the relations presented in an earlier section. Conceptually, Johnson's spreadsheet could be extended to calculate such estimates based on the number of independent realizations obtained in the data acquisition process.

Since the calculations are based on a uniform-displacement uncertainty, uncertainty estimates near surfaces will be underestimated. For example, Stanislas et al. (2005) found discrepancies from instantaneous displacements of about 0.5 pixels near the wall and about 0.2 pixels further away. The near-wall discrepancies may be systematic (i.e., bias uncertainties).

\section{CONCLUDING REMARKS}

The purpose of the fluid dynamics experiments in the MIR flow system at INL is to develop benchmark databases for the assessment of CFD solutions of the momentum equations, scalar mixing, and turbulence models for typical VHTR plenum geometries in the limiting case of negligible buoyancy and constant fluid properties. One aspect of the complex flow in a prismatic VHTR is being addressed: flow and thermal mixing in the lower plenum (the "hot streaking" issue). The flow in the lower plenum can locally be considered to be a situation of multiple jets into a confined crossflow with obstructions. Flow is expected to be turbulent with momentum-dominated turbulent jets entering; buoyancy influences are estimated to be negligible in normal full-power operation. Experiments are needed for the combined features of the lower plenum flows. Missing from the typical jet experiments available are interactions with nearby circular posts and vertical posts in the vicinity of vertical walls, with both near-stagnant surroundings at one extreme and significant crossflow at the other.

The objective of the present report is to develop understanding of the magnitudes of experimental uncertainties in the results to be obtained in such experiments. The experiments will use optical techniques, primarily PIV, in the INL MIR flow system. Unheated MIR experiments are first steps when the geometry is complicated. One does not want to use a computational technique that will not even handle constant properties properly. The benefit of the MIR technique is that it permits optical measurements that determine flow characteristics in passages and around objects to be obtained without locating a disturbing transducer in the flow field and without distorting the optical paths. With a transparent model of a different refractive index than the working fluid, the optical rays can be refracted in such a manner that measurements are either impossible to perform or require extensive, difficult calibrations.

Many definitions and approaches are employed in obtaining quantitative estimates of uncertainties in experimental results. As emphasized by Kline (1985a), "an uncertainty is not the same as an error. An error in measurement is the difference between the true value and the recorded value. An uncertainty is the possible value that the error might take on in a given measurement," as well as one can estimate. Since the true value is generally unknown in realistic experiments, the values of the errors are also unknown; accordingly, the term "error" is not appropriate for reporting the quantitative accuracy of an experiment. Recent practice in reporting uncertainties categorizes them as "precision" or "bias." Earlier descriptions included random error or repeatability for "precision" uncertainty and fixed or systematic error for "bias" uncertainty.

Uncertainties in PIV velocity measurements include those introduced during recording of multiple exposures (such as ones introduced by distortion of the scene being recorded), limited lens and film resolution, three-dimensional effects, bias introduced by large velocity gradients, and inaccuracies due to processing algorithms. Guezennec and Kiritsis (1990) addressed the PIV velocity error as functions of 
image noise level, number of particles, and particle displacement. Willert (1992) examined dependencies on particle density, particle displacement, and velocity gradients. Many investigators have examined processes and algorithms for estimating the particle displacement in PIV images.

A useful series of comparative studies and consequent reports is provided by the International PIV Challenges. Global agreement between the different methods was considered to be good. One key conclusion was that synthetic images were not fully representative of real images. The aim of Case B in their second challenge was to assess the performance of PIV/PTV algorithms for a synthetic turbulent flow with strong velocity gradients. In an ideal evaluation of PIV results, which analyzed synthetic particles generated from a DNS database, Stanislas et al. (2005) found discrepancies from instantaneous displacements of about 0.5 pixels near the wall and 0.2 pixels further away.

As noted by Melling (1997), the accuracy of the velocity field determination is ultimately limited by the ability of the scattering particles to follow the unsteady motion of the continuous phase. The particles in the MIR VHTR experiment are silver-coated, hollow glass spheres with a nominal mean particle diameter of about $14 \mu \mathrm{m}$ with $90 \%$ less than $21 \mu \mathrm{m}$, and their density is estimated as $1.65 \mathrm{~g} / \mathrm{cc}$. The density ratio then is about 2. Using the approximations of Mei with kinematic viscosity of about $14 \mathrm{cS}$ for the oil and a radius of $10.5 \mu \mathrm{m}$, one finds the estimated cutoff frequency to be above $40 \mathrm{kHz}$. The frequency of shedding from the posts in the MIR model is estimated to be on the order of one $\mathrm{Hz}$, and, therefore, the particles should follow the flow. An alternate approach to examine the question of particle motion is in terms of settling velocity (or terminal velocity due to gravity). The terminal velocity for a small particle falling through a stagnant fluid due to gravitational forces can be estimated via a force balance for steady motion. For our particles, this estimate gives about $0.02 \mathrm{~mm} / \mathrm{s}$ or less and $\mathbf{R e}_{\mathbf{p}}$ about $3 \times 10^{-5}$. The lowest flow velocity we might encounter would probably be about $1 \mathrm{~cm} / \mathrm{s}$. Thus, in this sense, we again would expect the particles to follow the flow adequately for the PIV.

Because the basic measurement of a PIV system is an instantaneous velocity component deduced from the translation $\Delta \mathbf{x}_{\mathbf{p}}$ of a group of particles over a time interval $\Delta t$,

$$
V_{p}=\frac{\Delta x_{\text {Pixel }}}{\Delta t}
$$

where the subscript Pixel indicates a measurement in pixels of the recorded image, the basic percent uncertainty in the velocity can be represented as

$$
\left(\% U n c V_{p}\right)=\sqrt{\left(\% U n c \Delta x_{\text {Pixel }}\right)^{2}+(\% U n c \Delta t)^{2}}
$$

The propagation of such uncertainties into the mean statistics reported then depends on the extent to which the uncertainties are random (precision) or systematic (bias) and the manner of presentation of results.

Studies with LaVision approaches show that, in idealized situations, with twenty particles in an interrogation area of $32 \times 32$ pixels, bias and rms errors vary from about 0.025 to 0.1 pixels for displacements $\Delta \mathbf{x}_{\text {Pixel }}$ of one to ten pixels. With the IA $=16 \times 16$ pixels and 200 particles, these uncertainties fall in a range of about 0.1 to 0.2 pixels up to $\Delta x$ of 7 , and then they explode. The processing algorithm for the LaVision PIV system gives uncertainties in displacements of about 0.05 pixels for synthetic images. For images of real experiments, additional factors involved include calibration, focus, displacement of particles, particle seeding, spatial gradients within each interrogation spot, image contrast, operator settings, etc. Therefore, a value of 0.05 pixels probably can be considered to be about the best possible under ideal circumstances. 
For the LaVision PIV system, the random uncertainty in the timing is estimated to be about $1 \mathrm{~ns}$. For the typical pulse separation of $100 \mu$ s (or more) used for the MIR PIV, the uncertainty would be $\leq 0.001 \%$ and therefore negligible compared to the displacement uncertainty.

In three-dimensional operation, the laser sheet thickness may be determined from measuring a correlation peak once a self-calibration procedure is completed. For the current operations, this thickness is estimated to be $2 \pm 0.2 \mathrm{~mm}$. For the current experiment, it is estimated that a point in an image in the vertical (x-y) plane may be determined to within about $0.11 \mathrm{~mm}(0.0043 \mathrm{in}$.) by using the cursor and image magnification. Thus, a relative location between two points within the same image view may be determined to about $2.5 \times 0.11 \approx 0.16 \mathrm{~mm}(0.006 \mathrm{in}$. $)$.

With the current MIR model and the 3D-PIV system, McIlroy and colleagues (2006) examined the effects of sample size for a typical set of measurements, using an approach comparable to that of Uzol and Camci (2001). About 10\% of the vectors under the jets and at the bottom of the plenum in the slower region were labeled invalid by LaVision software, while, in the middle of the plenum, about $30 \%$ or more were invalid. This approach evaluates effects of random uncertainties on the mean statistics for this particular situation (view and processing). Trends were generally as observed by Uzol and Camci. Preliminary conclusions were that, for this view and processing parameters, about 750 images should be collected to reduce the scatter in mean-velocity statistics to between 0.4 and $10 \%$ for velocities greater than about $2 \mathrm{~m} / \mathrm{s}$.

In addition to the uncertainties in velocity and turbulence measurements by PIV algorithms, other experimental uncertainties must be estimated for proper assessment of proposed CFD codes. These uncertainties include, but are not limited to, the geometry of the model, the properties of the working fluid, flow rates, and, consequently, Reynolds numbers.

Other uncertainties that have not yet been addressed in this treatment include near-wall quantities and calibration or parallax uncertainties. The calibration problems affect the local value of the scale factor $\mathrm{S}$ and the perceived location of the measurement. They can be expected to vary with position but presumably are essentially fixed at any one position.

In many experiments it is important to determine the local wall shear stress; in the process, the effective location of the surface may also be determined relative to the sampling grid. For a turbulent wall flow, this determination normally requires multiple mean-velocity measurements within the so-called linear layer near the wall $\left(\mathrm{y}^{+}<\sim 5\right)$ and the deduction of the mean-velocity gradient from them. Whether such an approach is feasible in the current experiment is not yet clear. The uncertainties in velocity gradients depend on uncertainties in both the mean velocities and in the spatial distances over which the gradients are evaluated. As noted earlier, Stanislas et al. (2003) found discrepancies from instantaneous displacements of about 0.5 pixels near the wall and 0.2 pixels further away. The high velocity gradients near the surface may also introduce excessive gradients across the interrogation window, giving a bias to the gradient deduced. Further studies are needed for specific cases in order to develop quantitative understanding of this situation. 


\section{REFERENCES}

Abernethy, R. B., R. P. Benedict, and R. B. Dowdell, 1985. ASME measurement uncertainty. J. Fluids Eng., 107, pp. 161-164.

Adrian, R. J., 1988. Statistical properties of particle image velocimetry measurements in turbulent flow. Laser anemometry in fluid mechanics, Lisbon: LADOAN Inst. Superior Technico, pp. 115-129.

Adrian, R. J., 1991. Particle-imaging techniques for experimental fluid mechanics. Ann. Rev. Fluid Mech., $\underline{23}$, pp. 261-304.

Bassett, A. B., 1888. Treatise on hydrodynamics, vol. II. London: Deighton, Bell and Co.

Becker S., C. M. Stoots, K. G. Condie, F. Durst, and D. M. McEligot, 2002. LDA-measurements of transitional flows induced by a square rib. J. Fluids Engr., 124, pp. 108-117.

Budwig, R. S., H. M. McIlroy, W. J. Dalling, D. M. McEligot, K. G. Condie, R. J. Pink, and G. E. McCreery, 2001. Near-wall behavior of a transitional boundary layer. Amer. Physical Soc. Fluid Dynamics meeting, San Diego, November. Bull. APS, 뜨, No. 10, p. 198.

Condie, K. G., G. E. McCreery, H. M. McIlroy, and D. M. McEligot, 2005. Development of an experiment for measuring flow phenomena occurring in a lower plenum for VHTR CFD assessment. Technical report INL/EXT-05-00603, 21 September.

Corino, E. R., and R. S. Brodkey, 1969. A visual observation of the wall region in turbulent flow. J. Fluid Mech., 37, pp. 1-30.

Crepeau, J. C., H. M. McIlroy, S. Reese, G. E. McCreery, K. G. Condie, D. M. McEligot, Y. G. Guezennec, R. S. Brodkey, and R. Clarksean, 1998. Fluid mechanic studies relating to drying and passivation in an idealized SNF canister. ANS 3rd Topical Meeting on Spent Nuclear Fuel and Fissile Material Management, Charleston, S. C., September.

Cui, M. M., and R. J. Adrian, 1997. Refractive index matching and marking methods for highly concentrated solid-liquid flows. Exp. Fluids, 22, pp. 261-264.

Durst, F., J. Jovanovic, and J. Sender, 1993. Detailed measurements of the near wall region of turbulent pipe flows. Proc., 9th Symp. Turb. Shear Flows, Kyoto, paper 2-2.

Durst, F., Keck, T., and Kleine, R., 1979. Turbulence quantities and Reynolds stress in pipe flow of polymer solutions measured by two-channel laser-Doppler anemometry. Proc., 6th Symp. on Turbulence, Rolla, Mo.

Forliti, D. J., and P. J. Strykowski, 2005. Controlling turbulence in a rearward-facing step combustor using countercurrent shear. J. Fluids Eng., 127, pp. 438-448.

Forliti, D. J., P. J. Strykowski, and K. Debatin, 2000. Bias and precision errors of digital particle image velocimetry. Exp. Fluids, 28 , pp. 436-447.

Guezennec, Y., and N. Kiritsis, 1990. Statistical investigation of errors in particle image velocimetry. Exp. Fluids, 10, pp. 138-146.

Halliday, D., and R. Resnick, 1970. Fundamentals of physics. New York: John Wiley.

Huang, H., D. Debri, and M. Gharib, 1997. On errors of digital particle image velocimetry. Meas. Sci. Technol., $\underline{8}$, pp. 1427-1440.

Johnson, R. C., 2006. Uncertainty in PIV measured displacements. Letter report, INL, 8 August.

Keane, R. D., and R. J. Adrian, 1990. Optimization of particle image velocimeters. Part I: Double pulsed systems. Meas. Sci. Technol., 1, pp. 1202-1215. 
Keane, R. D., and R. J. Adrian, 1992. Theory of cross-correlation analysis of PIV images. Appl. Sci. Research, 49, pp. 191-215.

Kim, W., J. Sung, J. Y. Yoo, and M. H. Lee, 2004. High-definition PIV analysis on vortex shedding in the cylinder wake. J. Visualization, 7, No. 1, pp. 17-24.

Kline, S. J., 1985a. The purposes of uncertainty analysis. J. Fluids Eng., 107, pp. 153-160.

Kline, S. J., 1985b. 1983 Symposium on Uncertainty Analysis closure. J. Fluids Eng., 107, pp. 181-182.

Kline, S. J., and F. A. McClintock, 1953. Describing uncertainties in single-sample experiments. Mech. Engng., 75, No. 1, pp. 3-8.

Kreplin, H.-P., 1973. Eine Methode zur Linearisierung von Heissfilmsignalen mit dem Digitalrechner PDP-15 und ihre Anwendung bei Messungen in einer turbulenten Kanalströmung. Report 2/1973, Max Planck Institut für Strömungsforschung, Göttingen. Diplomarbeit, Uni. Göttingen.

Kreplin, H.-P., 1976. Experimentelle Untersuchung der Längsschwankungen und der wandparallelen Querschwankungen der Geschwindigkeit in einer turbulenten Kanalströmung. Mitt. no. 63/1976, Max Planck Institut für Strömungsforschung und AVA Göttingen. Ph.D. dissertation, Uni. Göttingen.

LaVision, 2002. DaVis software seminary; Working with PIV systems. LaVision GmbH, D-37081 Göttingen, 6-8 November.

Lourenco, L., and A. Krothapalli, 1995. On the accuracy of velocity and vorticity measurements with PIV. Exp. Fluids, $\underline{18}$, pp. 421-428.

Luff, J. D., T. Drouillard, A. M. Rompage, M. A. Linne, and J. R. Hertzberg, 1999. Experimental uncertainties associated with particle image velocimetry (PIV) based algorithms. Exp. Fluids, 26, pp. 36-54.

MacDonald, P. E., J. W. Sterbentz, R. L. Sant, P. D. Bayless, R. R. Schultz, H. D. Gougar, R. L. Moore, A. M. Ougouag, and W. K. Terry, 2003. NGNP preliminary Point Design -- Results of the initial neutronics and thermal-hydraulic assessments. Tech. report INEEL/EXT-03-00870 Rev. 1, INEEL.

McCreery, G. E., K. G. Condie, R. L. Clarksean, and D. M. McEligot, 2002. Convective processes in spent nuclear fuel canisters. Heat Transfer 2002 (Twelfth International Heat Transfer Conference, Grenoble, August), Vol. 4, pp. 663-668.

McCreery, G. E., R. J. Pink, K. G. Condie, and D. M. McEligot, 2003. Fluid dynamics of ribbed annuli. NuReTH-10, Seoul, Oct.

McCreery, G. E., R. J. Pink, K. G. Condie, W. J. Dalling, D. M. McEligot, K. J. Knight, and G. L. McAllister, 2001. Computational and physical modeling collaboration for DARPA Chem/Bio program application. Tech. rpt. INEEL/EXT-2001-1397, INEEL.

McEligot, D. M., and fourteen others, 2005. Advanced computational thermal fluid physics (CTFP) and its assessment for light water reactors and supercritical reactors. Final tech. rpt. INL/EXT-05-00901, 31 October.

McEligot, D. M., and McCreery, G. E., 2004, Scaling studies and conceptual experimental designs for NGNP CFD assessment, Technical report INL/EXT-04-02502, 30 November.

McEligot, D. M., and H. Eckelmann, 2006. Laterally converging duct flows: Part 3. Mean turbulence structure in the viscous layer. J. Fluid Mech., 549, pp. 25-59.

McIlroy, H. M., 2006. The effect of sample size on experimental accuracy of ensemble-averaged PIV measurements. Letter report, INL, 10 August.

McIlroy, H. M., and R. J. Pink, 2005. KNERI model location study results. Letter report, INL, 1 August. 
McIlroy, H. M., and R. J. Pink, 2006. Laser traverse stage and laser head alignment. Letter report, INL, 20 April.

McIlroy, H. M., D. M. McEligot, G. E. McCreery, K. G. Condie, and R. J. Pink, 2006. Experimental measurement of flow phenomena in a VHTR lower plenum model. Presented at ANS 2006 Annual Meeting, Reno, 4-8 June.

Mei, R., 1996. Velocity fidelity of flow tracer particles. Exp. Fluids, 22, pp. 1-13.

Melling, A., 1997. Tracer particles and seeding for particle image velocimetry. Meas. Sci. Technol., 8, pp. 1406-1416.

Metzger, M. M., 2002. Scalar dispersion in high Reynolds number turbulent boundary layers. Ph. D. thesis, Univ. Utah.

Moffat, R. J., 1982. Contributions to the theory of single-sample uncertainty analysis. J. Fluids Eng., 104, pp. 250-260.

Moffat, R. J., 1985. Using uncertainty analysis in the planning of an experiment. J. Fluids Eng., 107, pp. 173-178.

Moffat, R. J., 1988. Describing uncertainties in experimental results. Exp. Thermal Fluid Sci., 1, pp. 3-17.

Orr, B., E. Thomson, and R. S. Budwig, 1998. Drakeol 5 thermophysical property measurements. Project report, Mechanical Engineering Department, Univ. Idaho, 18 December 1997; revised 15 January 1998.

Parker, J., and P. Merati, 1996. An investigation of turbulent Taylor-Couette flow using laser Doppler velocimetry in a refractive index matched facility. J. Fluids Engr., 118, pp. 810-818.

Prasad, A. K., R. J. Adrian, C. C.. Landreth, and P. W. Offutt, 1992. Effect of resolution on the speed and accuracy of particle image velocimetry interrogation. Exp. Fluids, $\underline{13}$, pp. 105-116.

Raffel, M., C. E. Willert, and J. Kompenhans, 1998. Particle image velocimetry: a practical guide. Berlin: Springer.

Rood, E. P., and D. P. Telionis, 1991. Editorial on experimental uncertainty. J. Fluids Eng., 113, September, pp. 313-314.

Roth, G. I., and J. Katz, 2001. Five techniques for increasing the speed and accuracy of PIV interrogation. Meas. Sci. Technol., 12, pp. 238-245.

Roth, G. I., D. T. Mascenik, and J. Katz, 1999. Measurements of the flow structure and turbulence within a ship bow wave. Phys. Fluids, 11, pp. 3512-3523.

Sabersky, R. H., A. J. Acosta, E. G. Hauptmann, and E. M. Gates, 1999. Fluid flow, 4th edition. Upper Saddle River, N. J.: Prentice Hall.

Shuster, J. M., R. J. Pink, D. M. McEligot, and D. R. Smith, 2005. The interaction of a circular synthetic jet with a cross-flow boundary layer. AIAA paper 2005-4749, AIAA Fluid Dynamics Conference, Toronto, June.

Smith, R. E., and S. Wehofer, 1985. From measurement uncertainty to measurement communications, credibility and cost control in propulsion ground test facilities. J. Fluids Eng., 107, pp. 165-172.

Soranna, F., Y.-C. Chow, O. Uzol, and J. Katz, 2006. The effect of inlet guide vanes wake impingement on the flow structure and turbulence around a rotor blade. J. Turbomachinery, 128, pp. 82-95.

Sridhar, G., and J. Katz, 1995. Drag and lift forces on microscopic bubbles entrained in a vortex. Phys. Fluids, $\underline{7}$, pp. 389-399. 
Stanislas, M., K. Okamoto, and C. J. Kähler, 2003. Main results of the First International PIV Challenge. Meas. Sci. Technol., 14, pp. R63-R89.

Stanislas, M., K. Okamoto, C. J. Kähler, and J. Westerweel, 2005. Main results of the Second International PIV Challenge. Exp. Fluids, 39, pp. 170-191.

Taylor, J. R., 1982. An introduction to error analysis. Mill Valley, Cal.: Univ. Science Books.

Taylor, J. R., 1997. An introduction to error analysis, 2nd ed. Sausalito, Cal.: Univ. Science Books.

Ullum, U., J. J. Schmidt, P. S. Larsen, and D. R. McCluskey, 1998. Statistical analysis and accuracy of PIV data. J. Visualization, 1, No. 2, pp. 205-216.

Uzol, O., and C. Camci, 2001. The effect of sample size, turbulence intensity and the velocity field on the experimental accuracy of ensemble averaged PIV measurements. PIV'01 paper 1096, 4th Int. Symp. On Particle Image Velocimetry, Göttingen, 17-19 September.

Westerweel, J., 2000. Theoretical analysis of the measurement precision in particle image velocimetry. Exp. Fluids (Suppl.), pp. S3-S12.

Westerweel, J., 2000b. Effect of sensor geometry on the performance of PIV interrogation. Laser techniques applied to fluid mechanics (Ed.: R. J. Adrian et al.), Berlin: Springer, pp. 37-55.

Willert, C., 1992. The interaction of modulated vortex pairs with a free surface. Ph.D.. thesis, Univ. California San Diego.

Zukauskas, A., 1972. Heat transfer from tubes in cross flow. Adv. Heat Transfer, $\underline{8}$, pp. 93-160. 